\title{
Evaluation of Phase-Diversity Techniques for Solar-Image Restoration
}

\author{
Richard G. Paxman and John H. Seldin \\ Electro-Optics Laboratory, Environmental Research Institute of Michigan, \\ P.O. Box 134001, Ann Arbor, MI 48113-4001 \\ Mats G. Löfdahl and Göran B. Scharmer \\ Royal Swedish Academy of Sciences, Stockholm Observatory, \\ S-13336 Saltsjöbaden, Sweden \\ and \\ Christoph U. Keller \\ National Solar Observatory, National Optical Astronomy Observatories, \\ P.O. Box 26732, Tucson, AZ 85726-6732
}

\begin{abstract}
Phase-diversity techniques provide a novel observational method for overcomming the effects of turbulence and instrument-induced aberrations in ground-based astronomy. Two implementations of phase-diversity techniques that differ with regard to noise model, estimator, optimization algorithm, method of regularization, and treatment of edge effects are described. Reconstructions of solar granulation derived by applying these two implementations to common data sets are shown to yield nearly identical images. For both implementations, reconstructions from phase-diverse speckle data (involving multiple realizations of turbulence) are shown to be superior to those derived from conventional phase-diversity data (involving a single realization). Phase-diverse speckle reconstructions are shown to achieve near diffraction-limited resolution and are validated by internal and external consistency tests, including a comparison with a reconstruction using a well-accepted speckle-imaging method.
\end{abstract}

Subject headings: methods: observational, techniques: image-processing, Sun: granulation 


\section{Introduction}

An important goal in ground-based astronomy is to improve the angular resolution that can be achieved. The angular resolution is nearly always limited by phase aberrations introduced by atmospheric turbulence. For the special case of ground-based solar astronomy, the spatial resolution is typically limited to about $0^{\prime \prime} .5$ for short-exposure images $(\leq 20 \mathrm{~ms})$ and to about $1^{\prime \prime} 0$ for long-exposure images $(\approx 1 \mathrm{~s})$. Many basic processes on the sun, however, take place at scales below $1^{\prime \prime} 0$. The photon mean free path in the lower photosphere corresponds to about $0^{\prime \prime} 1$ at disk center. Magnetic structures may occur on even smaller scales. For example, magnetic elements outside of sunspots have typical diameters smaller than $0^{\prime \prime} 2$ (Keller 1995). Despite their small size, these small structures are believed to play an important role in large-scale phenomena such as the solar magnetic dynamo or irradiance variations. To understand a variety of solar phenomena, it is, therefore, indispensable to reach a spatial resolution well below the seeing limit and possibly approaching the diffraction limit of existing and future, large solar telescopes.

A number of sophisticated techniques have been conceived to combat the deleterious effects of atmospheric turbulence in astronomical imaging in general. Among these are speckle imaging, phase diversity, and phase-diverse speckle imaging. In this paper we evaluate the use in solar astronomy of phase-diversity and phase-diverse speckle, referred to jointly as phasediversity techniques. To undertake this evaluation, we have applied phase-diversity techniques to solargranulation data collected with the Swedish Vacuum Solar Telescope (SVST) on La Palma. A subset of these data was also processed with a conventional speckle-imaging method to demonstrate consistency between accepted and novel restoration techniques. Phase-diversity techniques are particularly attractive for solar astronomy because (1) they require relatively simple and inexpensive instrumentation, (2) they perform well with relatively few images in high-signal regimes, (3) they lead to a joint estimation of the object and the wavefront, and (4) they obviate the need for complicated calibration.

In the following section we summarize three relevant fine-resolution imaging techniques: conventional speckle imaging, phase diversity, and phase-diverse speckle. Phase-diversity techniques, including phase diversity and phase-diverse speckle, have been imple- mented differently by the Environmental Research Institute of Michigan (ERIM) group and researchers at the Stockholm Observatory that operate the SVST (referred to herein as the SVST group). These implementations are described in Section 3. Section 4 provides details of the data collection and processing. Results derived from applying phase-diversity techniques to various data subsets are presented in Section 5. A speckle restoration is also included in this section to provide an external reference. Conclusions regarding the suitability of phase-diversity techniques for solar imaging are drawn in Section 6 .

\section{Fine-Resolution Imaging Techniques}

Speckle imaging is a relatively mature technique for obtaining fine-resolution images in the presence of atmospheric turbulence. This technique requires the collection of many short-exposure images of a static object. The exposure time for each frame must be short enough that the evolving atmosphere can be regarded as frozen during the exposure. Clever processing of this short-exposure time series affords the restoration of fine-resolution information that would be irretrievable if a single, long-exposure image were collected (Dainty 1984). Speckle imaging requires the collection of many images (typically 100 or more) so that the ensemble average over the class of all possible realizations may be approximated by an arithmetic average over a finite number of realizations. Speckleimaging methods have been successfully adapted to the solar-imaging problem (Keller \& von der Lühe 1992, de Boer \& Kneer 1992, von der Lühe 1993. 1994).

Another technique whose aim is the restoration of fine-resolution images in the presence of phase aberrations is the method of phase diversity, first proposed by Gonsalves (1979, 1982). Högbom (1988) independently proposed a special case of this same technique. calling it the focal-volume method. Phase diversity requires the simultaneous collection of two (or more) short-exposure images. Typically, one of these images is the conventional focal-plane image that has been degraded by the unknown aberrations. A second image of the same object is formed by perturbing the unknown aberrations in some known fashion. This can be conveniently accomplished with a simple beam splitter and a second detector array that is translated along the optical axis. An image collected in this second optical channel will contain the effects 
of the unknown phase aberrations but will also be influenced by the intentional defocus, which adds a known quadratic phase. It is somewhat remarkable that estimates for the object and the unknown aberrations can be made from these two images, given the known quadratic phase diversity. The first use of the method of phase diversity to retrieve fineresolution solar images was recently reported (Löfdahl \& Scharmer 1994a,b).

Intuition suggests that in stressing regimes (eg. poor seeing or weak signal levels) a single pair of phase-diversity images may not contain enough information to estimate jointly and with high fidelity the object and wavefront. Even under favorable conditions for which phase-diversity is able to produce good wavefront estimates, we have observed that object information at isolated spatial frequencies may be irretrievably lost, resulting in object estimates with significant artifacts. These cases motivate a third fine-resolution imaging technique, referred to as phase-diverse speckle (Paxman, Schulz, \& Fienup 1992a, Paxman \& Seldin 1994). As its name suggests, phase-diverse speckle blends the fundamental concepts of phase diversity and speckle imaging. Phase-diverse speckle requires the simultaneous collection of one conventional short-exposure image and at least one short-exposure image with phase diversity, for each of multiple atmospheric realizations, as depicted in Figure 1. This makes for a relatively simple data-collection scheme. Fortunately, the primary strengths of the two constituent methods, namely the added information content in a sequence of shortexposure images and the wavefront identification provided by phase diversity, persist. Two different processing approaches have been demonstrated with real phase-diverse speckle data by Löfdahl and Scharmer (1994b), referred to here as partitioned phase-diverse speckle (PPDS, see section 3.2.) and by Seldin and Paxman (1994), called joint phase-diverse speckle (JPDS, see section 3.1.).

In order to draw precise distinctions between these fine-resolution imaging methods and to establish notation, we present our working data-collection model. We concentrate on the data-collection model for phasediverse speckle, from which it can be seen that phasediversity and speckle-imaging data sets are special cases. The incoherent isoplanatic image-formation process is well modeled by the following discrete convolution:

$$
g_{j k}(x)=\sum_{x^{\prime}} f\left(x^{\prime}\right) s_{j k}\left(x-x^{\prime}\right),
$$

where $f(x)$ is the object array, $s_{j k}(x)$ is an incoherent point spread function (PSF) corresponding to the $j$ th atmospheric realization and the $k$ th diversity channel, $g_{j k}(x)$ is the corresponding noiseless image, and $x$ is a two-dimensional coordinate. The size of the noiseless image array is determined by the field of view (FOV) of the detector, whereas the size of the object array should extend well beyond the FOV. Of course any detected images will contain noise. The detected data set is represented by $\left\{d_{j k}\right\}$, where

$$
d_{j k}(x)=\mathcal{N}\left[g_{j k}\right], \quad \begin{aligned}
j & =1,2, \cdots, J \\
k & =1,2, \cdots, K
\end{aligned} .
$$

and where the general noise operator $\mathcal{N}[\cdot]$ introduces photon noise, additive Gaussian noise, and/or any other noise sources that are appropriate. The data set contains image frames from a total of $J$ atmospheric realizations and $K$ diversity channels, where typically $K=2$. Phase diversity is introduced by including a known phase function in the system 's coherent transfer function (Goodman 1968).

$$
P_{j k}(u)=P(u) \exp \left\{i\left[\phi_{j}(u)+\theta_{k}(u)\right]\right\},
$$

where $P(u)$ is a binary function that serves as an appropriately scaled model of the telescope pupil function, $\phi_{j}(u)$ is an unknown phase-aberration function with contributions from the $j$ th atmospheric realization and the fixed telescope aberrations, $\theta_{k}(u)$ is a known phase-diversity function associated with the $k$ th diversity channel, and $u$ is the discrete spatialfrequency variable. The phase-diversity function $\theta_{k}(u)$, will be zero in the conventional channel and quadratic in the channel with defocus. It is convenient to parameterize the phase-aberration function using coefficients for an appropriate set of basis functions (such as Zernike polynomials):

$$
\phi_{j}(u)=\sum_{m=1}^{M} \alpha_{j m} \psi_{m}(u) .
$$

The incoherent PSF, $s_{j k}(x)$, is just the squared $m m$ ulus of the discrete Fourier transform (DFT) of the coherent transfer function in equation (3). Thus the noiseless image, $g_{j k}(x)$, implicitly depends upon b, h the object and aberration parameters.

The goal of phase-diverse speckle is to estimat. 1 he common object and each of the $J$ phase-aberratint 
functions (or equivalently the aberration parameters) from the $J K$ detected images, given the known phasediversity functions and the binary pupil function. Notice that this model accommodates conventional phase diversity when $J=1$. When $K=1$, the data set corresponds to conventional speckle data. Although conventional speckle processing seeks an object estimate, no attempt is made to estimate the individual phase-aberration functions associated with the atmospheric realizations.

\section{Implementation of Phase-Diversity Tech- niques}

Both the ERIM and SVST research groups have been working on phase-diversity techniques for several years. Although the data-collection paradigm and basic goals are common to both groups, the processing implementations differ, reflecting differing research paths, emphases, and insights. In this section we summarize the salient features of these differing implementations and quote references that provide details of the processing.

\subsection{ERIM Implementation}

A guiding philosophy of the ERIM group has been to model the forward problem (data collection) as accurately as possible and to use this model as the basis for solving the inverse problem (object and aberration estimation) using estimation-theoretic tools.

\subsubsection{ERIM noise model and likelihood function}

A Poisson noise model was selected because such a model accurately accommodates the combined effects of signal-dependent photon noise and additive Gaussian CCD readout noise. The number of photoconversions that occur at each detector element will be a Poisson-distributed random variable with a mean value prescribed by the noiseless image, $g_{j k}(x)$, given in units of mean detected photons per pixel. Although not explicitly shown here, an artificial bias is added to the noiseless image to model the readout noise (Snyder, Hammoud, \& White 1993). Assuming that the detected signal at each detector element is statistically independent, the probability of acquiring a data set $\left\{d_{j k}\right\}$, given the object and the aberration param- eters for each atmospheric realization, is given by

$$
\operatorname{Pr}\left[\left\{d_{j k}\right\}\right]=\prod_{j=1}^{J} \prod_{k=1}^{K} \prod_{x} \frac{g_{j k}(x)^{d_{j k}(x)} \exp \left(-g_{j k}(x)\right)}{d_{j k}(x) !} .
$$

We use the principle of maximum likelihood to solve the inverse problem. We jointly estimate the object and aberration parameters by maximizing the $\log$ of the likelihood function,

$$
L(\hat{f}, \hat{\alpha})=\sum_{j=1}^{J} \sum_{k=1}^{K} \sum_{x}\left[d_{j k}(x) \ln \widehat{g}_{j k}(x)-\widehat{g}_{j k}(x)\right],
$$

where a constant term that has no bearing on the maximization procedure has been dropped and the phase-aberration parameter estimates, $\hat{\alpha}_{j m}$, have been lexicographically arranged into a single vector, $\hat{\alpha}$. We use the caret symbol,. , throughout to indicate an estimate. Because aberration parameters for all $J$ realizations are estimated simultaneously along with the object parameters, we refer to reconstructions as joint phase-diverse speckle (JPDS) estimates.

\subsubsection{ERIM optimization algorithm}

The method of preconditioned conjugate gradients (Luenberger 1984), a conventional nonlinearoptimization technique, is employed to maximize equation (6) over the set of object pixel values and phase parameters. Conjugate-gradients optimization is an iterative procedure that, at each iteration, requires a single gradient computation and a line search involving repeated likelihood evaluations. A closed-form expression for the gradient of the log-likelihood fun:tion has been derived (Paxman, Schulz, \& Fienup) 1992b, Paxman \& Seldin 1994) and is used extensively in the iterative search.

\subsubsection{ERIM regularization}

The maximum-likelihood estimates of the objert pixels can be somewhat sensitive to noise and may require a regularization strategy in which resolution in the estimate is traded for noise suppression. Therr are many candidate regularization strategies. howeser in this implementation the method of sieves (Snytir \& Miller 1985) is employed. This is accomplisherl 1 y. constraining the object estimate to be of the form

$$
\hat{f}(x)=\sum_{x^{\prime}} \tilde{f}\left(x^{\prime}\right) v\left(x-x^{\prime}\right)
$$


which is the convolution of an artificial Poisson point process, $\tilde{f}(x)$, with a smoothing (or sieve) kernel, $v(x)$. The maximum-likelihood formulation remains unchanged. However, instead of estimating the object, we now estimate the new underlying process, $\tilde{f}(x)$. The final object estimate is formed from $\tilde{f}(x)$ and $v(x)$ using equation (7). The choice of an appropriate sieve is the subject of ongoing research, but the Gaussian kernel (Snyder \& Miller 1985) has been quite effective.

\subsubsection{ERIM treatment of edge effects}

In principle, the size of the FOV is limited by the extent of the detector array or a field stop. However, the $N \times N$ FOV over which the convolutional imaging model in equation (1) is valid will depend on anisoplanatic effects. We therefore treat an $N \times N$ subframe of data as an effective FOV (as if it were collected by a detector array of that extent), and we estimate the object-pixel values associated with these elements. In addition, we estimate object-pixel values within a guard band of width $B$ pixels surrounding the effective FOV. Although these guard-band pixels do not have a corresponding detector element within the effective FOV, they influence the data in two distinct ways. The first is through PSF sidelobes. For example, a bright object point in the guard band will create PSF sidelobes that spill into the effective field of view. The second mechanism derives from random image translations that occur as a result of differing tilt components among the atmospheric realizations in a phase-diverse speckle data set. Thus the main lobe of a PSF associated with an object point in the guard band may be directly sensed by detector elements within the effective FOV when the tilt component for a particular realization provides the right translation. The size of the guard band is selected so that the influence of pixels far from the effective FOV is negligible. This is related to the severity of the aberrations and the resulting PSF side-lobe structure and translations. The total number of object parameters is given by $(N+2 B)^{2}$.

Several aspects of the guard-band technique are appealing. The guard-band technique affords the accurate and efficient computation of the convolution in equation (1) using a DFT, or a fast Fourier transform (FFT) in practice. Although a DFT assumes a periodic object, estimated values for pixels at the outer rim of the guard band are allowed to "wrap around" since their influence on the estimated data is negligible. Another appealing aspect of the guardband method is that, unlike apodization techniques (Paxman \& Crippen 1990), the measured data are unperturbed. Finally, the guard-band method allows for the reliable retrieval of object pixels up to the edge of the detector-limited FOV, so long as the effective FOV is defined to abut the detector-limited FOV.

\subsection{SVST Implementation}

The purpose of the SVST-group implementation is to develop a fast and reliable method for obtaining nearly diffraction-limited images with the SVST in La Palma. The code has been operational since the spring of 1993 and is the first phase-diversity code used to demonstrate, through several consistency tests (Löfdahl \& Scharmer 1994a,b), that the technique works on real data. This is mainly due to the successful implementation of methods to deal with image boundary effects and for registration of focused and defocused images pairs.

\subsubsection{SVST noise models and metric}

The SVST code is based on two modifications of the error metric of Gonsalves and Chidlaw (1979). which relies on a Gaussian additive noise model. This model allows the estimation of the optimum object to be performed implicitly while the estimation of the optimum wavefront is done explicitly, which leads to a straightforward and computationally efficient code. However, the expression for the optimum object cannot be used directly because it leads to unlimited amplification of noise at spatial frequencies where the transfer functions of the focused and defocused images simultaneously approach very small values. This happens because the expression for the restored object gives a best fit to the data, including its noise. Of course what is needed is an expression for the restored object which is as accurate as possible, which means that the observed images must be filtered to reduce noise in the restored object. We have also found that high noise levels give slower convergence in the iterative determination of the wavefronts.

Our first modification to the error metric of Gonsalves and Chidlaw, therefore, is to introduce a noise filter, which is applied both to the observed focused and defocused images, in the expression for the error metric.

The second modification consists of accounting for the possibility that the signal-to-noise ratio (SNR) 
may be significantly different for the image channels, as in the case of beam-splitters that do not distribute light in equal proportions. With these two modifications the error metric becomes

$$
L_{j}=\sum_{u}\left|H_{j} D_{j 1}-\hat{F}_{j} \widehat{S}_{j 1}\right|^{2}+\gamma\left|H_{j} D_{j 2}-\widehat{F}_{j} \widehat{S}_{j 2}\right|^{2}
$$

where, in contrast to the ERIM formulation, the summation is in the Fourier domain rather than the image domain. $\widehat{S}_{j k}$ is an estimate of the optical transfer function (OTF), which is the Fourier transform of the estimated PSF. $H_{j}$ is the noise filter, and $\gamma$ is given by

$$
\gamma=\sigma_{1}^{2} / \sigma_{2}^{2}
$$

where $\sigma_{1}$ and $\sigma_{2}$ are the RMS values of the noise of the two channels. With $H_{j} \equiv 1$ and $\gamma=1$, the error metric of Gonsalves and Chidlaw (1979) is recovered. Following their derivation, which means estimating $F$ independently for each realization $j$, leads to an expression for the estimated Fourier object,

$$
\widehat{F}_{j}=H_{j} \frac{D_{j 1} \widehat{S}_{j 1}^{*}+\gamma D_{j 2} \widehat{S}_{j 2}^{*}}{\left|\widehat{S}_{j 1}\right|^{2}+\gamma\left|\widehat{S}_{j 2}\right|^{2}}
$$

where * used as a superscript denotes the complex conjugate. The corresponding error metric can be written as

$$
L_{j}=\sum_{u}\left|E_{j}\right|^{2}
$$

where the Fourier-domain error function is defined as

$$
E_{j} \equiv H_{j} \frac{D_{j 2} \widehat{S}_{j 1}-D_{j 1} \widehat{S}_{j 2}}{\sqrt{\left|\widehat{S}_{j 1}\right|^{2}+\gamma\left|\widehat{S}_{j 2}\right|^{2}}} .
$$

Earlier analysis (Löfdahl \& Scharmer 1994b) has shown that this method leads to good estimates of the wavefront parameters but that in poor seeing the restored objects are contaminated by artifacts from poor SNR at isolated spatial frequencies. These artifacts are removed by combining the results of two or more realizations to calculate the restored Fourier object in a fashion that is well known in the literature,

$$
\widehat{F}=H \frac{\sum_{j=1}^{J} D_{j 1} \widehat{S}_{j 1}^{*}+\gamma D_{j 2} \widehat{S}_{j 2}^{*}}{\sum_{j=1}^{J}\left|\widehat{S}_{j 1}\right|^{2}+\gamma\left|\widehat{S}_{j 2}\right|^{2}}
$$

where the OTFs must include the shifts necessary to bring all images into co-alignment. The filter $H$ is of similar form to the filters of individual realizations,
$H_{j}$, but refers now to several atmospheric realizations. These filters are specified in Section 3.2.3.

Because phase estimates derive from partitioned data whereas object estimates derive from these phase estimates in conjunction with a full phase-diverse speckle data set, we refer to this processing approach as partitioned phase-diverse speckle (PPDS).

\subsubsection{SVST optimization algorithm}

The expansion for $\phi_{j}$, equation (4), allows us to write $S_{j k}=S_{j k}\left(\alpha_{j m}\right)$, and therefore $E_{j}=E_{j}\left(\alpha_{j m}\right)$. Due to the nonlinear dependence of $E_{j}$ on $\alpha_{j m}$. the minimum has to be found iteratively from an initial estimate, usually $\phi_{j} \equiv 0$. This is implemented by approximating changes in $E_{j}$ by

$$
\delta E_{j} \approx \sum_{m} \frac{\partial E_{j}}{\partial \alpha_{m}} \delta \alpha_{j m}
$$

and seeking corrections to the coefficients, $\delta \alpha_{j m}$, such that the minimum of $L_{j}$ is found in the next iteration. This linearization results in a matrix equation of the type

$$
\mathbf{A} \cdot \delta \boldsymbol{\alpha}+\mathbf{b}=0
$$

where the elements of $\mathbf{A}$ and $\mathbf{b}$ are sums of different combinations of $E_{j}$ and its partial derivatives with respect to the aberration parameters (see Löfdahl \& Scharmer 1994b). These derivatives involve the transformed images, $D_{j k}$, the OTFs, $\widehat{S}_{j k}$, and the OTF derivatives, which are evaluated analytically. Note that $\mathbf{A}$ is an $M \times M$ matrix, where $M$ is the number of aberration parameters. This equation is solved with the singular value decomposition (SVD) method. as described in Section 3.2.3.

\subsubsection{SVST regularization}

In the SVST implementation, two methods of regularization (noise suppression) are employed: (1) the observed images are low-pass filtered and (2) the wavefront estimate is restricted by zeroing the least significant singular values of the system matrix, $\mathbf{A}$.

In order to provide noise reduction, the filters $H$, and $H$ must be specified. Since the main priority is to obtain good, restored images, it seems reasonable to choose $H$ such that the combined RMS error from noise and the filter is minimized in the restored objort. 
$\hat{F}$. This leads to

$$
H=1-\left\langle\left|N_{1}\right|^{2}\right\rangle\left\langle\frac{\sum_{j=1}^{J}\left|\widehat{S}_{j 1}\right|^{2}+\gamma\left|\widehat{S}_{j 2}\right|^{2}}{\left|\sum_{j=1}^{J} D_{j 1} \widehat{S}_{j 1}^{*}+\gamma D_{j 2} \widehat{S}_{j 2}^{*}\right|^{2}}\right\rangle,
$$

where $\langle\cdot\rangle$ denotes an expectation value. In practice, the second expectation value is estimated with a smoothing operation and the removal of noise peaks in the high frequency regime, see (Löfdahl \& Scharmer 1994b) for details.

The filter area expands automatically with the number of included realizations, as information is added in $\hat{F}$ at isolated frequencies and as the noise influence is reduced at high frequencies by the averaging of many realizations. The single-realization filters, $H_{j}$, are defined as the special case where the sum is over one realization.

Solving the matrix equation (15) directly for a large number of wavefront parameters gives poor convergence, or even divergence, in the iterative procedure and poor wavefront estimates. One conjecture is that this happens because the focused and defocused images do not contain enough information to distinguish between too many wavefront parameters. The matrix equation is therefore solved by means of the SVD method. The SVD method rearranges the equations, so that solutions are sought for orthogonal linear combinations of the parameters. These combinations are sorted in order of significance, as expressed by the singular values. Solving only the system of equations with singular values larger than a cutoff level, defined as a fraction of the largest singular value, restricts the solution to the subspace spanned by the most significant linear combinations, corresponding to the retained equations.

Recent experiments within the SVST group have shown that using a cutoff level of 0.02 permits Zernike parameters up through the 12 th radial degrees and azimuthal frequencies and generates wavefronts that conform better to Kolmogoroff covariance. This value of the cutoff level was found by trial and error to give only a $1 \%$ increase in the converged value for the error metric $L$. The SVD method eliminates the problem of over-parameterization in a simple and automatic way, independent of the type of object used to determine the wavefront parameters. It also significantly improves the convergence of the iterations, thus enhancing the speed of the code.

With the low-order wavefront expansion used for the current work, the inversion problem is well-conditioned.
We therefore used a cutoff of 0.0001 , which effectively de-activates the regularizing property of the SVD method.

\subsubsection{SVST treatment of edge effects}

The error metric of Gonsalves, as expressed in the Fourier domain, does not include the effects of boundaries of the images. Using FFTs to perform the convolutions needed for the calculation of the error functions and its derivatives produces severe wrap-around effects which can lead to very large errors in the derived wavefronts when using small sub-fields. This problem is avoided by transforming the error function and its derivatives to the image plane. Parseval's relation permits the summation in equation (11) to be calculated in the image domain. Observing that the wrap-around errors in the image-domain error function, $e_{j}$, are concentrated to the boundaries of the array, we use an array size that is large enough that the wrap-around effects are accommodated outside the effective FOV. The summation is then restricted to the FOV. Apodizing with a modified Hanning window function with a flat profile over the FOV further removes a high frequency pattern from the discontinuities at the array boundaries (Löfdahl \& Scharmer 1994b).

Like the ERIM algorithm, the technique affords the accurate and efficient computation of the convolution in equation (1) using FFTs. Furthermore, unlike pure apodization techniques (Paxman \& Crippen 1990), the measured data are unperturbed in the $N \times N$ area.

\section{Solar Data}

\subsection{Data Collection}

The data were collected with the $47.5 \mathrm{~cm}$ SVST in La Palma on April 27, 1993 (see Löfdahl \& Scharmer $1994 \mathrm{~b}$ for details). The multi-image, real-time frame selection system (see Scharmer \& Löfdahl 1991) monitored the fine-structure content in the focused channel and was set to select and store the best 100 image pairs out of 1500 recorded during 30 second intervals. The best seeing at the SVST usually occurs intermittently, so the series used for this analysis was recorded during 6 seconds of good seeing at 14:19 UT. This interval is short enough that no significant evolution of the granulation structure can take place. Observations were made through a $5.4 \mathrm{~nm}$ wide interference filter centered at $470 \mathrm{~nm}$. The known quadratic phase 
difference of the two image channels corresponds to a phase shift at the edge of the aperture equal to $0.985 \pm 0.06$ waves.

Images were recorded by two synchronized EEV video CCD cameras operating at $50 \mathrm{~Hz}(20 \mathrm{~ms}$ exposure time) and digitized to 8 bits by two Kontron DEC/IPS image processing systems. The image scale is 0.0706 and $0^{\prime \prime} 0732$ per pixel in the $x$ and $y$ directions respectively. (The mean value, $0^{\prime \prime} 0719$ was used for the inversions.) The image scales of the two image channels differ by less than $0.1 \%$, and the images are rotated by less than 0.1 degrees relative to each other.

\subsection{Preprocessing}

The frames in the two channels were corrected with their corresponding dark current and gain table calibrations. The gain table was determined by randomly moving the telescope and averaging a large number of frames. Since the bias level of the cameras changed with illumination, the bias was determined from covered parts of the CCD sensor and taken into account in the data reduction. Image-restoration techniques can be sensitive to small but consistent errors in the gain table. Despite the excellent results reported by Löfdahl and Scharmer (1994b), a thorough analysis of the sensitivity of phase-diversity restorations to camera calibration errors has not been undertaken. Therefore, a careful calibration procedure was followed here to mitigate the influence of calibration errors on our analysis. Following the procedure in (Keller, Stenflo, \& von der Lühe 1992), the gain table correction was improved by decomposing the Fourier transform of the average image into a high-frequency domain and a low-frequency domain. It is then assumed that the signal in the high-frequency domain is due to errors in the gain table only. The true average image may then be found with an appropriate low-pass filter. Each frame in the sequence is then corrected by multiplication with the ratio between the low-pass filtered and the original average frame according to

$$
d_{j k}^{\text {corrected }}=d_{j k} \frac{\sum_{j=1}^{100} \tilde{d}_{j k}}{\sum_{j=1}^{100} d_{j k}},
$$

where $\widetilde{r}$ represents the spatial low-pass filtering. The focused and defocused channels are treated separately.

Each defocused image was further adjusted so that its mean intensity was the same as its corresponding, focused image. A close inspection of the defocused images revealed a very weak, horizontal strip-pattern that could not be removed with the gain-table calibration. This pattern was removed in the following way: for each image the average column was determined by averaging along the horizontal direction. A highorder Legendre polynomial was subtracted from the average column to extract the high-frequency strip pattern and to remove any variations due to solar structures. This difference was then subtracted from every column in an image.

Shifts between consecutive frame pairs were determined via cross-correlation and then removed. The same shifts were applied to both channels, and only full pixel shifts were performed in order not to affect any high-spatial-frequency solar signal. Note that this prealignment was performed to accommodate the speckle reconstruction and is not required for phasediverse speckle. Finally, $128 \times 128$ pixel subframes were extracted in such a way that the average shift between focused and defocused subframes amounts to less than a pixel.

The entire sequence of 100 preprocessed image pairs is publically available and can be obtained by contacting the authors at the Stockholm Observatory.

\subsection{Processing}

The $128 \times 128$ subframes were selected with a innter region of $70 \times 70$ pixels that contains fine image details that are useful for assessing restoration fildity. This central region, or effective FOV, corresponds to a $5^{\prime \prime} 0 \times 5^{\prime \prime} 0$ patch, which is small enough to sat isfy an isoplanatic imaging assumption (Löfdahl \& Scharmer $1994 \mathrm{a}, \mathrm{b})$. Both the conventional speckle and SVSI group restorations use all the data in the larger 128 $\times 128$ subframes, but in both cases the restorations are most reliable over the center region due to "dge" effects. The ERIM restoration procedure does not us data outside the $70 \times 70$ center region, but the larger subframes were utilized in some cases to obtain a b.e. ter initial object estimate within the guard band of size $B=21$ pixels. All comparisons between restorations are made over this central area, and henreforth we will restrict our attention to this region.

Five examples out of the sequence of 100 pairs of preprocessed subframes are found in Figure 2. The top row contains conventional, focused images in $1 \mathrm{he}$ $5^{\prime \prime} .0 \times 5^{\prime \prime} .0$ central region, and the defocused couml. parts are found below. The RMS contrast ilefined 
here as the ratio of the standard deviation to the mean intensity) averaged over the entire sequence is $7.6 \%$ and $4.8 \%$ for the focused and defocused images, respectively. Examples of very good seeing are shown in Figures 2(a) and (b). Figure 2(c) is an average case, and (d) and (e) are examples of aberrated images that yield poor conventional phase-diversity restorations. It is interesting to note the dramatic effect of defocus on the image in (d).

To facilitate comparisons of restorations, both the ERIM and SVST groups parameterized the phase aberrations with Zernike polynomials 4 through 15 . From previous experience we knew that this level of wavefront parameterization leads to a well-conditioned inversion problem. The two tilt components, Zernike polynomials 2 and 3 , provide sub-pixel positioning of the estimated PSFs. For the case of JPDS, these parameters are included in the joint-estimation process. For PPDS, they are derived in post-processing by cross-correlating the phase-diversity object estimates. If the data were not prealigned, then the use of these polynomials would be even more critical. Another important parameter is the registration between the conventional and diversity channels. Typically, the ERIM approach is to estimate these parameters along with the aberrations and the object. However, to keep the implementations as similar as possible we decided to use predetermined values. The technique used here for estimating these parameters from the average of the data in the two imaging channels is discussed by Löfdahl and Scharmer (1994b). Other system parameters, such as the amount and the sign of diversity and the pixel spacing, were the same for both implementations as well.

\section{Results}

In this section we compare estimates made with: (1) ERIM phase-diversity techniques, (2) SVST-group phase-diversity techniques, and (3) conventional speckle imaging. We show that the two implementations of phase diversity yield virtually identical object and aberration estimates, but that these object estimates are susceptible to artifacts, indicating a clear need for phase-diverse speckle estimation. We show quantitatively that JPDS is slightly better than PPDS. The internal consistency of the phase-diverse speckle estimates is demonstrated by analyzing estimates derived from disjoint data sets, and external validation is obtained by comparing these restorations with the speckle restoration. Finally, the influence of anisoplanatism is considered.

\subsection{Implementation Invariance for Phase Di- versity}

The ERIM and SVST group's approaches to phasediversity techniques are quite distinct. The noise model, estimator, optimization algorithm, regularization, and edge treatment are some of the ways in which the techniques differ. Despite these differences, we have discovered strong evidence to indicate that in most cases the different implementations yield, for all practical purposes, identical solutions. The first evidence of this is presented here for the case of conventional phase-diversity; i.e., restoration from a single pair of images.

Figure 3 contains the conventional phase-diversity object estimates derived from each of the 5 pairs of images in Figure 2. The data in Figure 2 were selected because the restorations derived from them offer a nice comparison of the two implementations. The first trend to note in Figure 3 is that the ERIM restorations in the top row have slightly higher spatial frequency content than the SVST group's restorations in the bottom row, which is a direct consequence of the different approaches to object regularization. The attempt to restore more fine detail can have the $1 \mathrm{nn}$ desirable side-effect of boosting high-frequency artifacts, creating a slightly mottled appearance. Typically, when the SVST group's noise filter is applied to an ERIM restoration, the resulting estimate is vit sually indistinguishable from the SVST restoration Thus, the overall features in the restorations from the two implementations are often quite similar. but the SVST group's restorations appear to be low-pass filtered versions of the ERIM estimates.

A useful measure of similarity between two istimates, $\hat{f}_{1}(x)$ and $\hat{f}_{2}(x)$, is via the normalized RMS error, $\epsilon$, defined via

$$
\epsilon^{2}=\frac{\frac{1}{N^{2}} \sum_{x}\left[\hat{f}_{1}(x)-\hat{f}_{2}\left(x-x_{r}\right)\right]^{2}}{\left[\frac{1}{N^{2}} \sum_{x} \hat{f}_{1}(x)\right]^{2}},
$$

where $x_{r}$ brings $\hat{f}_{2}$ into registration with $\hat{f}_{1}$, and areraging is done over $N^{2}$ pixels. The misregistration $x_{r}$, is estimated to sub-pixel accuracy by interpolating the peak of the cross-correlation of the two estimates. Phase-diversity estimates were made for the entire sequence of 100 image pairs, and the average error between the two implementations is $i=1 . i$ 
with a standard deviation of $1.0 \%$. Figures $3(\mathrm{a})$ - (c) contain estimates for which the match is very good, with errors of $0.8 \%, 1.2 \%$, and $1.2 \%$, respectively. Figure $3(\mathbf{a})$ is an example of a high-quality phasediversity restoration because it compares favorably with phase-diverse speckle restorations presented in subsequent sections (see Figure 8). Visual cues can be taken from the central portion of the restorations, where there is a narrow intergranular lane and a small, bright feature at the tip of the arrow. This small feature is slightly brighter in the ERIM restoration, reflecting a higher concentration of energy due to the recovery of higher spatial frequencies. On the other hand, mottle can be observed on some of the larger granules. When the SVST-group noise filter for each of the restorations in Figures 3(a) - (c) is applied to the corresponding ERIM restoration, $\epsilon$ drops to $0.4 \%$, $0.9 \%$, and $1.0 \%$, respectively, the mottle disappears, and the differences in the restorations become visually imperceptible.

The restorations in Figures 3(b) and (c) degrade somewhat with respect to (a), but what is also notable is that the ERIM and SVST group's restorations share the same features, even when they are false. For example, the small features near the narrow intergranular lane at the center of the image are smeared into nearby granules in both of the restorations in (c). Referring back to Figure 2(c), it is clear why these features, which are blurred severely in the original data, are not fully recovered. Careful inspection of Figures 3(a) - (c) reveals that, despite differences in spatial frequency content, features such as granule shape and intensity variations along the granule edges are virtually identical.

Greater differences between implementations are observed in Figures 3(d) and (e), with $\epsilon=4.0 \%$ and $2.1 \%$, respectively. The SVST group restoration is superior to the ERIM restoration in (d), particularly with respect to the large granule in the lower left. Conversely, the ERIM restoration in (e) does not display the stripe artifact that runs at an angle through the SVST restoration. Neither of these restorations is particularly good, however. The trend observed across the 100 restorations is that large discrepancies between implementations are observed rarely and only for cases when the blurring is too severe for conventional phase diversity to be effective.

The other major component of phase-diversity restoration is the estimation of the phase aberration. In Figure 4 we show 12 scatter plots of Zernike coef- ficient estimates from the two implementations for polynomials 4 through 15 . In each sub-graph the coefficients estimated by the two implementations for each of the 100 image-pairs are scattered about the line $y=x$. We note immediately the high correlation between the aberrations derived from the two implementations. Aside from one or two outliers, the aberration estimates are very consistent. One measure of agreement is formed by taking the square root of the mean over the pupil of the average, squared wavefront difference (averaged over 100 realizations). This measure of RMS difference between implementations is a negligible 0.043 wave. This is well below the well-known Marechal aberration-tolerance condition of $1 / 14$ th wave RMS phase error (Born \& Wolf 1980). Systems that meet the Marechal condition are considered to be well-corrected, producing imagery for which the degradation would be difficult to perceive. Thus, the aberration estimates from the two implementations yield point-spread functions that are visually indistinguishable.

\subsection{Partitioned vs. Joint Estimation in Phase- Diverse Speckle}

Aside from implementation invariance, important conclusions to draw from the results presented in Figure 3 are that even an above-average conventional phase-diversity restoration like the one in (c) still suffers from residual blur, and that in some cases the restorations are dominated by artifacts. This observation was made by Löfdahl and Scharmer (1994b) and led to a partitioned phase-diverse speckle (PPDS) estimation strategy (Section 3.2.1.) in which pairs of restorations were combined to produce much higher quality object estimates. Similarly, Seldin and Paxman (1994) demonstrated that restorations from the same data using a joint phase-diverse speckle (JPDS) algorithm (Section 3.1.1.) became better and more consistent as image pairs were added. From these results it is clear that there is a distinct advantage to using phase-diverse speckle instead of conventional phase diversity. Given the need for phase-diverse speckle, a natural question to address is whether there is an advantage to using the joint-estimation approach as opposed to a partitioned technique.

As with conventional phase diversity, we find that object estimates derived from the JPDS and PPDS algorithms are virtually identical, with a negligible difference of $\epsilon=0.3 \%$ when the entire sequence of 100 image pairs is used. Not surprisingly, the JPDS and 
PPDS restorations are also visually identical, as observed in Figures $8(\mathrm{~b})$ and (c), respectively. Intuition suggests that JPDS would provide better estimates of both the object and the aberrations because the ratio of the number of measurements to parameters increases significantly with the addition of each new pair of aberrated images of the same object. However, the JPDS and PPDS restorations are so similar for these data that we are left to wonder if there is a distinct advantage to a joint-estimation approach in this regime.

The object estimates alone do not tell the whole story, and so the accuracy of the phase aberration estimates must also be considered. Given two aberration estimates, one from conventional phase diversity and the other from JPDS, we seek to quantify the accuracy of each. To do this without the aid of a simulation, we generate an estimated point-spread function, $\hat{s}_{j k}$, from each wavefront, convolve it with the corresponding object estimate, and compare the resulting image estimate to the measured image. We define a fidelity metric for the $j$ th wavefront estimate, $\widehat{\phi}_{j}$, in a normalized mean-squared error sense as

$$
R^{2}\left(\hat{\phi}_{j}\right)=\frac{\frac{1}{N^{2}} \sum_{k} \sum_{x}\left[d_{j k}(x)-\hat{g}_{j k}(x)\right]^{2}}{\left[\frac{1}{N^{2}} \sum_{x} \hat{f}(x)\right]^{2}}
$$

where averaging is done over $N^{2}$ pixels, and $\widehat{g}_{j k}$ is formed from $\hat{f}$ and the estimated point-spread function, $\widehat{s}_{j k}$, which is a function of $\widehat{\phi}_{j}$, via the convolution

$$
\hat{g}_{j k}(x)=\sum_{x^{\prime}} \hat{f}\left(x^{\prime}\right) \hat{s}_{j k}\left(x-x^{\prime}\right) .
$$

Note that the numerator of equation (19) is identical (aside from a sign change) to the log-likelihood function for phase diversity under an additive Gaussian noise model (Paxman, Schulz, \& Fienup 1992b). Smaller values of $R$ imply a better match of the object and aberration estimates to the data. We evaluate $R\left(\widehat{\phi}_{j}\right)$ for $j=1,2, \cdots, 100$ for both the ERIM JPDS wavefronts estimates and the SVSTgroup phase-diversity wavefront estimates. When evaluating $R$ for the JPDS aberration estimates, the 100 -realization JPDS restoration in Figure $8(\mathrm{~b})$ is used for $\hat{f}$. Similarly, the SVST-group conventional phase-diversity aberrations estimates are evaluated using the 100-realization PPDS object estimate in Figure 8(c).

Figure 5 is a scatter plot of $R\left(\hat{\phi}_{j}\right)$ for PPDS versus $R\left(\widehat{\phi}_{j}\right)$ for JPDS. The mean fidelity metric for the JPDS wavefronts is $1.3 \%$, compared with $1.5 \%$ for phase diversity. Since the object estimates used to generate the fidelity metric differ by only $0.3 \%$, we can conclude that most of the error is due to sources other than the object. In 97 of 100 cases, the fidelity metric for the JPDS aberration estimate is less (better) than the wavefront derived with conventional phase diversity, and the fidelity metrics are very close for the other 3 cases. There is a fairly strong linear correlation that indicates that the rise and fall in fidelity metric tracks fairly well for the two cases. Nonetheless, it is clear that there are 15 to 20 cases for which the phase-diversity fidelity metric is significantly worse in a relative sense. In an absolute sense we are observing only very small improvements in the aberration estimates, which improves the fidelity metric by only a fraction of a percent. Apparently, phase-diversity wavefront estimates are so good in this regime that there is little room for improvement when using JPDS. Even so, it remains necessary to use multiple realizations to obtain a good object estimate via PPDS. The reason for this is simply that the OTF for any one realization tends to reach very low values at isolated spatial frequencies which vary from realization to realization. This explanation is consistent with the ringing artifacts found in Figures $3(\mathrm{~d})$ and (e). The fact that the JPDS wavefront estimates are almost always better than the PPDS counterparts shows that the jointestimation approach is a successful concept which is likely to be superior to PPDS in different imaging regimes; e.g. reduced signal levels, smaller isoplanatic patches, stronger aberrations, more aberration parameters, etc.

\subsection{Internal Consistency of Phase-Diverse Speckle Estimates}

Both conventional phase diversity and phase-diverse speckle imaging have been investigated extensively with past simulation studies. These simulations are an important component in establishing the redibility of phase-diversity image restoration. An even more important step was taken by using real solar granulation data to demonstrate the consistency of aberration estimates from neighboring image patches (Löfdahl \& Scharmer 1994b). A measure of internal consistency with respect to object estimates was demonstrated with the same data set for the case of JPDS (Seldin \& Paxman 1994) applied to a small number of realizations. In this case several JPDS 
restorations were made using different sets of images collected within seconds of each other, and these restorations were shown to be highly consistent with each other. We explore this in more detail here using many more realizations, to further demonstrate the consistency of object estimates.

Object estimates are a random process with a mean and a variance, and any one restoration is a sample drawn from such a process. Like other random processes, the mean of an object estimate can be estimated using a sample mean derived from independent trials. The same is, of course, true for estimating the variance. We define our trials as restorations of the same object with different input data. For example, we formed a conventional phase-diversity restoration for each of the 100 image pairs, and from these 100 restorations we computed a sample mean and variance. We also formed samples of 2-realization JPDS restorations by partitioning the sequence of 100 pairs into 50 disjoint sets, containing $J=2$ image pairs each. The same partition of the sequence into disjoint sets was done for the cases of $J=5,10,25$, and 50 realizations, and restorations were made for every set. Examples of one restoration for each of the 6 cases investigated are shown in Figure 6 . The 1- and 2-realization restorations in Figures 6(a) and (b), respectively, have visible artifacts, but restoration quality is quite good in the 5 -realization case in Figure $6(\mathrm{c})$. It is difficult to see much variation across the 10-, 25-, and 50-realization restoration examples in Figures 6(d) - (f), respectively.

To quantify the stability of the JPDS estimates as a function of the number of realizations, we consider the sample variance about the sample mean. This is a measure of internal consistency: if the variance of restorations formed from disjoint sets of data decreases as the number of realizations increases, then we conclude that restorations become more consistent with more realizations. If this variation is also quite small, then we can also conclude that any random false detail is too small to be considered problematic. A common method for measuring the variance of an estimator about its mean is via the coefficient of variation (Frieden 1983), the square of which is defined at each pixel as

$$
c_{v}^{2}(x)=\frac{1}{L} \frac{\sum_{\ell=1}^{L}\left[\hat{f}_{\ell}(x)-\bar{f}(x)\right]^{2}}{f^{2}(x)},
$$

where $\hat{f}_{\ell}$ is the $\ell$ th sample from a set of $L(=100 / J)$,
$J$-realization restorations, and

$$
\vec{f}(x)=\frac{1}{L} \sum_{\ell=1}^{L} \widehat{f}_{\ell}(x)
$$

Figure 7 plots the spatial average of $c_{v}(x)$, denoted by $\bar{c}_{v}$, as a function of the number of realizations. By averaging over the pixels, we characterize the variation about the mean at a pixel with a single number. The error bars on this plot represent the confidence in $\bar{c}_{v}$ to within one standard deviation. The standard deviation of $\bar{c}_{v}$ was computed as the square root of the variance of equation (21), which takes into account the correlation of $c_{v}(x)$ from pixel to pixel. We note that Figure 7 displays a monotonic behavior from which we conclude that estimates become more consistent as realizations are added. Also, the greatest gains in consistency are made for small numbers of realizations, and $\bar{c}_{v}$ is less than $1 \%$ for $J=10$. Beyond this point the gains are small, supporting our visual assessment of the sample restorations in Figure 6 . Figure 7 does not indicate how the mean images change as a function of number of realizations. The RMS error between any pair of mean images has an average of about $\epsilon=0.3 \%$, which reflects a high consistency for the expected restoration regardless of the number of realizations used.

\subsection{External Consistency of Phase-Diverse Speckle Restorations}

In the previous section we cited past evidence and presented new results that confirm the internal consistency of phase-diverse speckle object estimates. External validation of phase aberration estimates from conventional phase diversity has been demonstrated via the fixed aberrations of the SVST. These aberrations were estimated over time as the SVST turret position changed and were shown to evolve according to theoretical predictions (Löfdahl \& Scharmer 1994b). Speckle imaging provides another independent way to validate the object estimates. For this work, the Fourier amplitudes have been reconstructed with the Labeyrie (1970) method, whereas the phases have been estimated with the Knox and Thompson (1974) technique. The Fourier amplitudes were calibrated with a model of the Earth's atmosphere by Korff (1973). Fried's (1966) parameter, the only free parameter of the atmospheric model, was estiniated with the spectral ratio technique (von der Lühe 198.4) to be $r_{o}=18.5 \mathrm{~cm}$. A detailed description of the $\mathrm{im}$ - 
plementation of the above-mentioned techniques has been given by von der Lühe (1993).

Figure $8(\mathrm{a})$ contains the speckle reconstruction, which should be compared with the 100-realization phase-diverse speckle estimates from JPDS and PPDS in (b) and (c), respectively. Note that the overall features are identical in all 3 restorations, including subtle intensity variations along the edges of some granules. There is no evidence of artifactual details in either of the phase-diverse speckle restorations. In fact, the speckle restoration appears to be slightly smoother, with less high-frequency detail. To reinforce the important point that 100 image pairs are many more than required for phase-diverse speckle, we show examples of JPDS and PPDS 5-realization restorations in Figures $8(\mathrm{~d})$ and (e), respectively. It is clear that excellent restorations can be obtained with a factor of 20 fewer realizations than used for (b) and (c). Because a reliable speckle reconstruction like that in (a) could not be obtained with so few images, phase-diverse speckle can be viewed as an important method for improving both the spatial and the temporal resolution of ground-based observations.

The restorations in Figures 8 (b) and (c) differ from the speckle restoration in (a) with an error of $\epsilon=2.0 \%$ and have an error of only $\epsilon=0.3 \%$ between them. Some of the disparity is due to differences at high spatial frequencies, but we have found that small differences exist at low spatial frequencies as well. Figure 9 plots the radially-averaged power spectra of the 3 restorations. Each spectrum was formed by first applying a Hanning window to the restoration, performing a 2-D Fourier transform, and then taking the magnitude-squared. After appropriate scaling, each spectrum was integrated over annuli in the spatial-frequency domain. As expected, the JPDS and PPDS spectra are very consistent out to $80 \%$ of the diffraction-limited cutoff frequency, and differences beyond this point can be attributed to differing regularization techniques. We note that the power in the speckle reconstruction begins to depart from the others at about half of the cutoff frequency, and that the speckle reconstruction appears to have approximately $30 \%$ more power than the others up to this point.

Defining contrast as the ratio of the standard deviation to the mean, we find that the contrast of the speckle restoration is higher $(12.6 \%)$ than either of the phase-diverse speckle estimates (11.0\%). One plausible explanation for this could be due to an insufficient representation of the phase aberrations with only the first 15 Zernike polynomials. It has been demonstrated in simulations that with a zonal (pixel-bypixel) parameterization of the pupil, the full contrast of the object can be recovered (Seldin, Paxman, \& Elste 1995). These same simulations consistently underestimated the contrast when using only the first 15 Zernike polynomials. On the other hand, the speckle reconstruction relies on a model for the atmospheric turbulence and does not account for fixed telescope aberrations. So the possibility of an underlying model mismatch remains, and the correctness of the restored contrast remains an open issue that is best studied with controlled experiments. Despite this significant difference in contrast, we conclude that the phasediverse speckle estimates are consistent with and provide finer detail than the reconstruction obtained with an accepted speckle-imaging technique.

\subsection{Evidence of Anisoplanatism}

Space-variant blur is encountered when objects $e x-$ tend beyond the isoplanatic patch associated with the intervening atmosphere. The imaging model used here is space-invariant, and any anisoplanatism should degrade the quality of the restorations. Phasediverse speckle object reconstructions are susceptible to geometric distortions associated with anisoplanatism because of the underlying assumption of a time-invariant object across all realizations. Furthermore, intuition suggests that JPDS wavefront timates, unlike PPDS wavefront estimates, could also be hurt by these inter-realization geometric distortions. The fact that both PPDS and JPDS succond so well here is due in part to the selection of an appropriately-sized image patch, which was guided ly previous analysis of this data sequence by Löfdahl and Scharmer (1994b).

Despite the successful restorations obtained with these images, there is still evidence of anisoplanat $1: 111$ in this sequence. To characterize the geometrir listortion, each image, $d_{j k}$, in the sequence was resam. pled (destretched) to match the estimated imagr. $\hat{I}_{j} k$ (eq. [20]), formed from the 100-realization JPISS ...timate in Figure 8(b). The destretching for the jth realization is estimated by computing local corrilations between $d_{j k}$ and $\widehat{g}_{j k}$ on a $3 \times 3$ segmentaliun of the images for both the conventional and diwersity channels. The 9 shifts obtained from the $3 \times 3$ gril if correlations are averaged over the two channels. and a coordinate transformation that defines the destrin. h- 
ing function is formed by interpolating between the average shifts at the center of each segment. The degree of distortion, or image warping, can be summarized with a single number

$$
w=\sqrt{x_{d}^{2}+y_{d}^{2}}
$$

where $x_{d}$ and $y_{d}$ are the RMS shifts in the two directions computed over all segments. Thus, large values of $w$ reflect large deviations from a global shift and a higher degree of warping across the image.

Figure $10(\mathrm{a})$ plots $w$ (in units of pixels) versus the fidelity metric, $R$, for each of the 100 JPDS phase aberration estimates. There is a clear correlation (0.74) between $R$ and the amount of distortion - realizations with the worst distortion yield the worst fidelity metric. In contrast, there is no apparent correlation between the RMS of the estimated aberrations and $R$, indicating that the strength of the turbulence is not the primary source of poor estimates. A new sequence of destretched images, $\tilde{d}_{j k}$, was created, and the fidelity metric was recomputed via equation (19) using $\tilde{d}_{j k}$ in place of $d_{j k}$. Note that the same phase aberration estimates were used to compute the "destretched" fidelity metric, and that the most improvement in this metric occurs for the cases with large distortion. Remarkably, the improvement in the average fidelity metric after destretching is 3 times greater than the corresponding improvement when moving from conventional phase diversity to phasediverse speckle. Figure 10(b) also suggests that aberration estimates are not hurt by the joint-estimation approach at this level of anisoplanatism. Although the improvement in the fidelity metric is only a fraction of a percent, we conclude that all phase-diversity techniques would benefit from the accommodation of anisoplanatic effects, particularly in the presence of stronger turbulence. An obvious approach would be to apply JPDS or PPDS to destretched data. Alternatively, parameters in an anisoplanatic-imaging model could be directly estimated from the original data (Paxman, Thelen, \& Seldin 1994).

\section{Conclusions}

In this investigation, we have evaluated phasediversity techniques, including two implementations of these techniques, and provided credibility for the scientific utility of these relatively novel observational techniques for solar astronomy. Using data collected with only modest instrumentation, we have recon- structed near diffraction-limited images of solar granulation.

\subsection{Comparison of Implementations}

The ERIM and SVST implementations of phasediversity techniques have been compared. In spite of significant differences in noise model, estimator, optimization algorithm, regularization, and edge treatment, the phase-diversity estimates were found to be virtually identical. The invariance of estimates under differing noise models can be understood by considering the similarities of the Poisson and additive Gaussian noise models for this application. Poisson noise is proportional to the square root of the intensity at each detector element. In addition, when the intensity is sufficiently large, the cumulative distribution function for each Poisson random variable is wellapproximated by a normal distribution (Feller 1968). For low-contrast images such as aberrated and/or defocused images of solar granulation, the Poisson noise will be approximately constant across the image, thus resembling additive Gaussian noise. In fact, our reconstructions suggest that the Gaussian noise model is entirely adequate for imaging solar gramulation However, the Poisson model is the more general of the" two for describing photo-detection events. and it can also be expanded to accommodate additive (iaussian noise sources, such as CCD readout noise. A Poisson model may be important when using low-noise rameras to image high-contrast scenes such as sunspot umbrae with umbral dots or for photon-limited imaging scenarios such as narrow-band solar imaging or nighttime astronomy.

An advantage of the Gaussian noise model is that it leads to an optimization search within a reduceddimension parameter space. The resulting estimation algorithm, as currently implemented by the SIST group, requires significantly fewer operations than the current ERIM algorithm, when operating with a sufficiently small number of aberration parameters. II The SVST algorithm computations are dominated by Fourier transform calculations. The total number of FFTs required to perform a phase-diversity reronstruction is $I \cdot(7+4 M)$, where $I$ is the number of it erations. For the data analyzed here, iterations wer" stopped when the RMS of the change of the wavr. front was less than $10^{-3}$ radians, which is quite inll. servative. This stopping criterion led to an average if about $I=12$. Quality reconstructions are obtalli..l if iterations stop when the metric changes less lian 
$0.5 \%$, giving $\langle I\rangle=4.7$. With $M=12$ and $I=5$, it takes $275 J$ FFTs to process $J$ realizations with the SVST program.

The ERIM algorithm is also dominated by twodimensional FFTs. Approximately $(3 V+4) J K$ FFTs are computed per iteration, where $V$ is the number of likelihood evaluations made during the conjugategradient line search. Because this expression is independent of the number of aberration parameters, $M$, there is no additional computational cost per iteration for a fine parameterization of the wavefront. For the data analyzed herein, $V=10$ and typically the number of iterations performed was $I=60$. These processing criteria are very conservative. We have since shown that visually indistinguishable object estimates are produced when relaxing the line search to $V=5$ and stopping the iterations when the metric changes by $0.02 \%$, giving approximately $I=40$. Using $V=5$, $I=40$, and $K=2$ we see that the ERIM code requires approximately $1520 \mathrm{~J}$ FFTs, considerably more than is required for the SVST code. In cases with $M$ sufficiently large, such as a zonal aberration parameterization (i.e.using pixels in the pupil), the ERIM code probably has a computational advantage, owing to the independence of the FFT count per iteration on $M$. It should be remembered that neither of the algorithms has been truly optimized with respect to computational efficiency.

Another implementation issue is whether estimates are made in a joint or a partitioned fashion. Object estimates were found to be virtually identical using JPDS and PPDS. However, JPDS aberration estimates were found to be slightly more consistent with the data than were the PPDS estimates. Although the small improvement in aberration estimates produced by JPDS is of little practical value in the reconstructions shown, it does suggest that a jointestimation strategy could be of value when imaging in the presence of stronger turbulence or in regimes of reduced signal strength. We also believe that improvement due to joint estimation is currently limited by anisoplanatic effects that vary from realization to realization and by the under-parameterization of wavefronts. Both ERIM (Seldin, Paxman, \& Elste 1995) and SVST groups have found evidence that 15 Zernikes is an under-parameterization of the wavefronts, but the optimal number of wavefront parameters remains an open issue.

When comparing the two approaches to treating edge effects, we see that both methods effectively han- dle problems associated with objects that extend beyond the FOV. The ERIM guard-band method allows for the reliable retrieval of object pixels up to the edge of the detector-limited FOV, providing more efficient use of camera pixels and rendering a method for utilizing interesting phenomena recorded along the camera borders.

\subsection{Comparison of Techniques}

Although estimated wavefronts were found to be essentially the same for all phase-diversity techniques. our results reiterate that phase-diverse speckle object estimates are significantly better than phase-diversity estimates. This can be understood by considering the OTFs involved. The OTFs for any one realization tend to reach very low values at isolated spatial frequencies, and the inversion of single-realization data can give false detail at these spatial frequencies. However, these troublesome spatial frequencies vary from realization to realization so that when multiple realizations are used, the likelihood of restoring false detail is dramatically diminished. Furthermore, we have quantitatively shown that estimates become increasingly more consistent as the number of realizations is increased. The added value of additional realizations can only increase when anisoplanatism is accommodated.

Conventional speckle imaging can also be compared with phase-diverse speckle. Speckle imaging has the hardware advantage that only a single speckle camera is required. On the other hand, simple optical designs have been implemented that put both diversity images on a single camera, at the expense of reduced FOV. Speckle imaging also offers a computational advantage, requiring only $J$ FFTs of size $N^{2}$. Phase-diverse speckle has an important advantage in that excellent object reconstructions can be derived from a relatively few $(J \approx 5)$ realizations, whereas speckle imaging requires a much larger number $(J \approx 100)$ of realizations. With fewer realizations required, phase-diverse speckle can more readily be used for time-series observation of the evolution of solar phenomena. Whereas conventional speckle requires a separate speckle calibration step that relies on an atmospheric model (von der Lühe 1993) that normally doesn't accommodate fixed telescope aberrations, phase-diverse speckle requires no such step and is not hurt by fixed aberrations. In fact, because phase-diverse speckle estimates individual phase aberration realizations, these estimates can be averaged 
to determine fixed aberrations (Löfdahl \& Scharmer 1994b).

\subsection{Future Directions}

Although there are issues which deserve greater study, including optimal wavefront parameterization, the effects of anisoplanatism, and the effects of atmospheric evolution during an exposure time, we conclude that phase-diversity techniques are sufficiently mature to be used routinely for solar observations. A new phase-diversity beam-splitter that puts two diversity channels on a single large $C C D$, giving each a useful FOV of approximately $700 \times 1000$ pixels, has recently been installed at the SVST. This is available as a common-user instrument. Excellent data, consisting of time sequences (several hours in duration) of active regions, have already been obtained. In addition, scientific data, consisting of images of bright points, pores, sunspots, plages, and network magnetic fields in white-light, $H \alpha$, and $\mathrm{MgIb}_{2}$ that cover up to several hours have been recorded with a multi-camera system at the Vacuum Tower Telescope at Sacramento Peak. Preliminary analysis of both data sets confirm our expectation that phase-diverse speckle opens a new observational window, providing excellent spatial and temporal resolution over extended periods of time

We are also in the process of migrating phasediverse speckle methods to faint-object regimes, including narrow-band observations in solar astronomy and nighttime astronomical observations. As an example, we have recently used phase-diverse speckle to successfully correct for residual aberrations in an adaptive-optics system when imaging binary stars (Seldin, Paxman, \& Ellerbroek 1995).

We are grateful for the encouragement and helpful comments provided by $G$. Elste and $O$. von der Lühe. We also acknowledge the many useful discussions with B. Thelen. RGP and JHS were supported by the NASA Space Physics Program Office, NASA Grant No. NAGW-4069, which is gratefully acknowledged. MGL was supported by the Swedish Science Research Council. The Swedish Vacuum Solar Telescope is operated on the island of La Palma by the Royal Swedish Academy of Sciences in the Spanish Observatorio del Roque de los Muchachos of the Instituto de Astrofísica de Canarias. CUK was supported by the Swiss National Science Foundation under Grant No. 8220-037202. The National Optical
Astronomy Observatories are operated by the Association of Universities for Research in Astronomy, Inc. (AURA) under cooperative agreement with the National Science Foundation.

\section{REFERENCES}

de Boer, C. R. \& Kneer, F. 1992, A\&A, 264, L24

Born, M. \& Wolf, E. 1980, Principles of Optics (New York, NY: Pergamon Press), 468

Dainty, J. C. 1984, in Topics in Applied Physics, Vol 9, Laser Speckle and Related Phenomena, ed. J. C. Dainty (New York: Springer), 255

Feller, W. 1968, An Introduction to Probability Theory and Its Applications, Volume I (New York, NY: John Wiley \& Sons)

Fried, D.L. 1966, JOSA, 56, 1372

Frieden, B.R. 1983, Probability Statistical Optics and Data Testing (New York: Springer-Verlag)

Goodman, J. W. 1968, Introduction to Fourier Optics (New York: McGraw Hill)

Gonsalves, R. A. \& Chidlaw, R. 1979, in Proc. Sor. Photo-Opt. Instrum. Eng. 207: Applications of Digital Image Processing III, ed. A. G. Tescher. 32

Gonsalves, R. A. 1982, Opt. Eng., 21, 829

Högbom, J. A. 1988, in Proc. of the 10th Sacramento Peak Summer: High Spatial Resolution Solar Observations, ed. O. von der Lühe, 166

Keller, C. U. \& von der Lühe, O. 1992, A\&A, 261. 321

Keller, C. U., Stenflo, J. O., \& von der Lühe, O. 1992, A\&A, 254, 355

Keller C.U. 1995, Reviews in Modern Astronomy. 8. 27

Knox, K. T. \& Thompson, B. J. 1974, ApJ, 193. L45

Korff D. 1973, JOSA, 63, 971

Labeyrie, A. 1970, A\&A, 6, 85

Löfdahl, M. G. \& Scharmer, G. B. 1994a, in Pror. of the 13th Sacramento Peak Summer Workshop 1992: Real Time and Post-Facto Solar Image (irrrection, ed. R. R. Raddick, 89 
Löfdahl, M. G. \& Scharmer, G. B. 1994b, A\&AS, 107, 243.

Luenberger, D. G. 1984, Linear and Nonlinear Programming (Reading, MA: Addison-Wesley)

von der Lühe, O., 1984, JOSA A 1, 519

von der Lühe, O. 1993, A\&A, 268, 374

von der Lühe, O. 1994, A\&A, 281, 889

Paxman, R. G. \& Crippen, S. L. 1990, in Proc. Soc. Photo-Opt. Instrum. Eng. 1351: Digital Image Synthesis and Inverse Optics, ed. A. F. Gmitro et al., 787

Paxman, R. G., Schulz, T. J., \& Fienup, J. R. 1992a, in Signal Recovery and Synthesis IV: Optical Society of America Technical Digest Series 11, 5

Paxman, R. G., Schulz, T. J., \& Fienup, J. R. 1992b, J. Opt. Soc. Am A, 9, 1072

Paxman, R. G., \& Seldin, J. H. 1994, in Proc. of the 13th Sacramento Peak Summer Workshop 1992 Real Time and Post-Facto Solar Image Correction, ed. R. R. Raddick, 112

Paxman, R. G., Thelen, B. J., \& Seldin, J. H. 1994, Opt. Lett., 19, 1072

Scharmer, G. B. \& Löfdahl, M. G. 1991, Adv. Space Res., 11, (5) 129

Seldin, J. H., \& Paxman, R. G. 1994, in Proc. Soc. Photo-Opt. Instrum. Eng. 2302: Image Reconstruction and Restoration, ed. T. J. Schulz \& D. L. Snyder, 268.

Seldin, J. H., Paxman, R. G., \& Eiste, G. H. 1995, Bulletin of the American Astronomical Society, 27, 956 .

Seldin, J. H., Paxman, R. G., \& Ellerbroek, B. L. 1995, in Adaptive Optics: Optical Society of America Technical Digest Series 23, 260

Snyder, D. L., \& Miller, M. I. 1985, IEEE Trans. Nucl. Sci., NS-32, 3864

Snyder, D. L., Hammoud, A. M., \& White, R. L. 1993, J. Opt. Soc. Am. A, 10, 1014

This 2-column preprint was prepared with the AAS IATEX macros v3.0. 
Fig. 1.- Data-collection scheme for phase-diverse speckle. A conventional short-exposure image and an additional short-exposure image that is intentionally defocused are simultaneously collected for each of multiple atmospheric realizations.

Fig. 2. - Examples of preprocessed data. Five realizations of conventional (upper) and diversity (lower) data are shown. Examples of good seeing are shown in (a) and (b). The image pair in (c) represents an average case, and (d) and (e) contain images from which conventional phase-diversity restorations are poor.

Fig. 3.- Examples of phase-diversity object estimates from ERIM (upper) and the SVST group (lower). The five restorations in (a) - (e) are derived from the corresponding data in Figure 2.

Fig. 4.- Scatter plots of Zernike coefficients of estimated aberrations using 2 phase-diversity implementations. The SVST and ERIM estimates for the entire sequence of 100 images for coefficients 4-15 are shown. The high correlation is strong evidence of implementation invariance.

Fig. 5.- Scatter plot (over realization) of the fidelity metric $R\left(\widehat{\phi}_{j}\right)$ for SVST-group phase diversity versus ERIM phase-diverse speckle. $R$ reflects the accuracy of the aberration estimates, and we observe that the joint phase-diverse speckle approach yields a better fidelity metric in 97 out of 100 cases.

Fig. 6.- Representative object restorations for different numbers of realizations. The restorations in (a) - (f) are derived using $J$ image pairs for $J=$ $1,2,5,10,25,50$, respectively. Note that we show only one of many candidate restorations for each value of $J$, but the same input image-pairs are always retained as $J$ is increased. The restorations are quite consistent for $J \geq 5$.

Fig. 7.- Spatially averaged coefficient of variation, $\bar{c}_{v}$, in the object estimate as a function of number of realizations, $J$. The error bars were computed using the variance of $\vec{c}_{v}$ and are one standard deviation above and below the estimated value.
Fig. 8.- Speckle and phase-diverse speckle restorations. (a) 100-realization speckle-imaging restoration; (b) 100-realization ERIM joint phase-diverse speckle restoration; (c) 100-realization SVST-group partitioned phase diverse speckle restoration; (d) 5realization ERIM restoration; (e) 5-realization SVSTgroup restoration.

Fig. 9.- Radially-averaged power spectra for speckle, ERIM joint phase-diverse speckle (JPDS), and SVST-group partitioned phase-diverse speckle (PPDS) restorations. The diffraction-limited cut off frequency has been normalized to unity.

Fig. 10.- Scatter plots of degree of distortion. " versus fidelity metric, $R$, before and after destretching of the data. (a) The fidelity metric for the 11101 ERIM phase-diverse speckle aberration estimates is correlated with the distortion; (b) After destretching the images, these same estimates yield fidelity merric values that are smaller on average and much less correlated with the original distortion. 

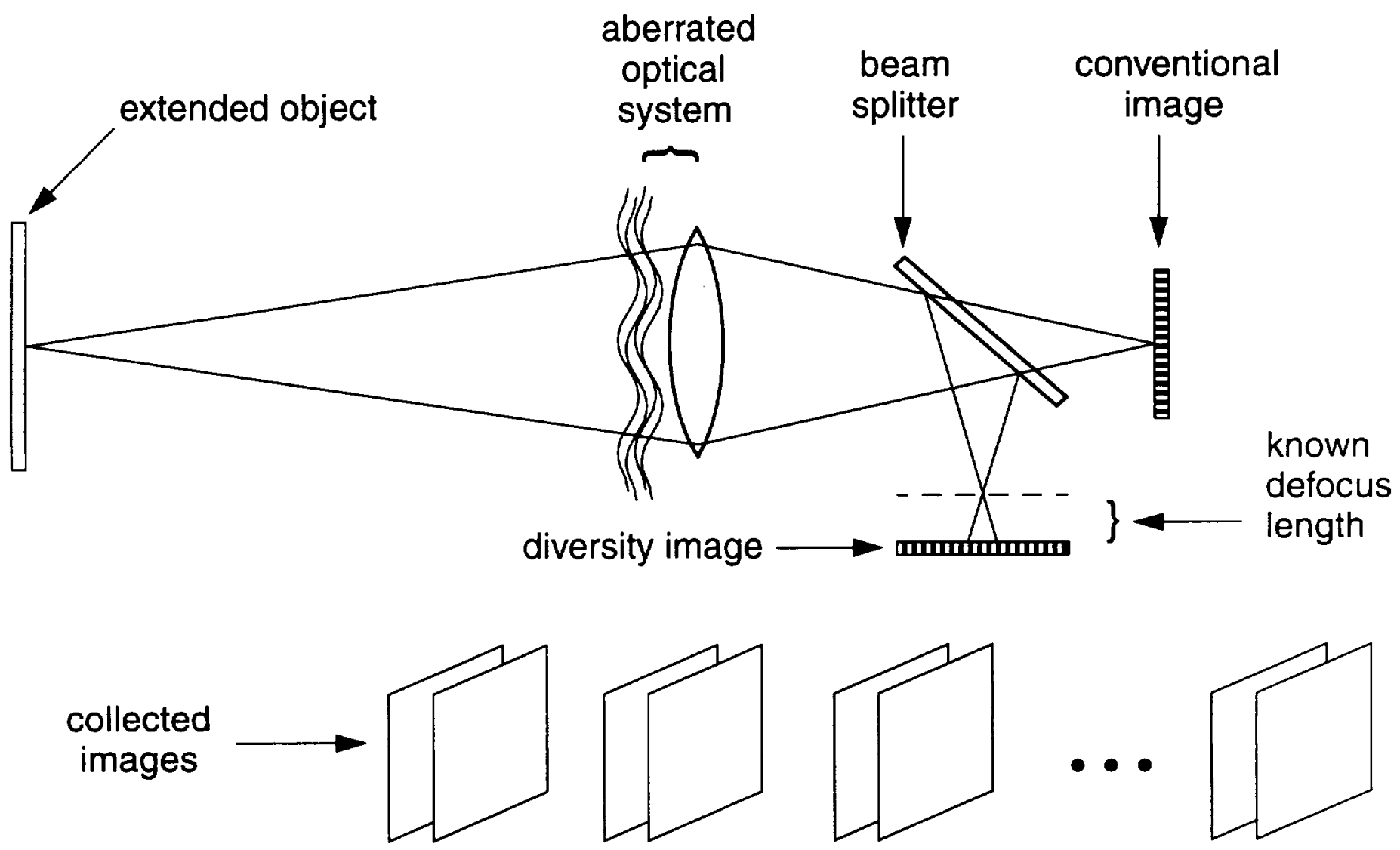
Figure 2
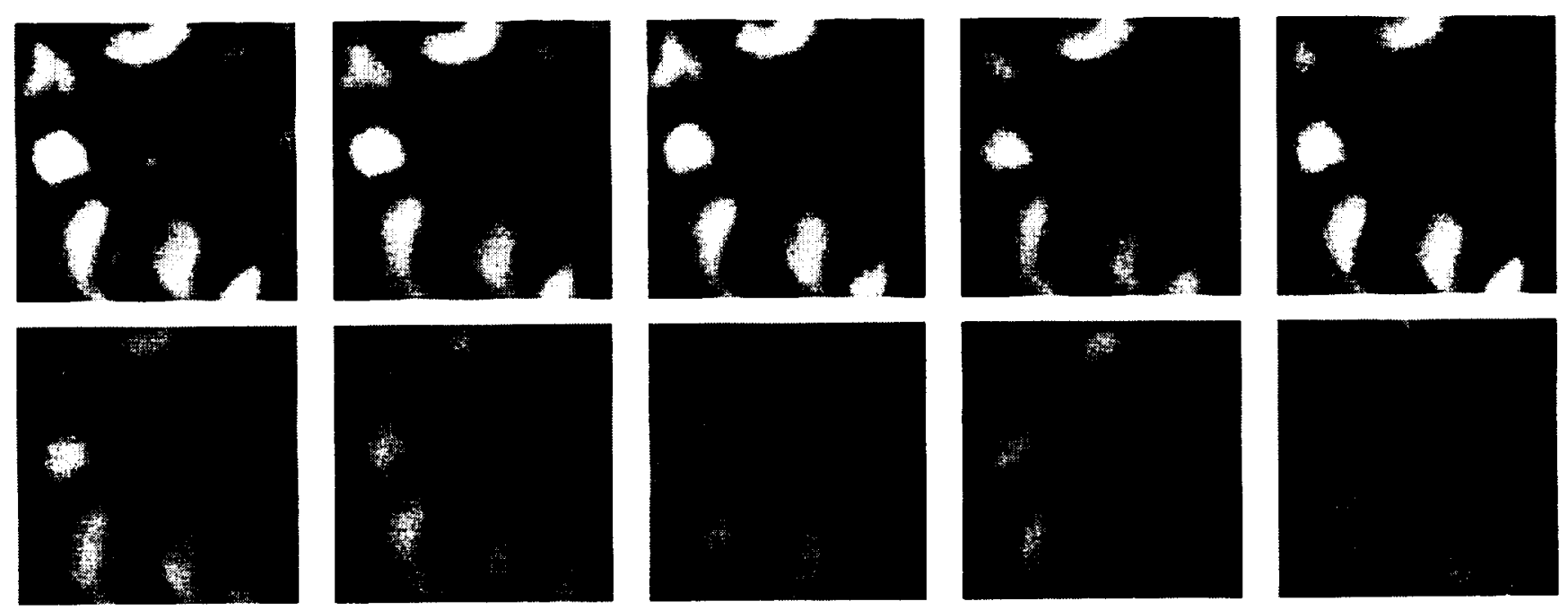

(a)

(b)

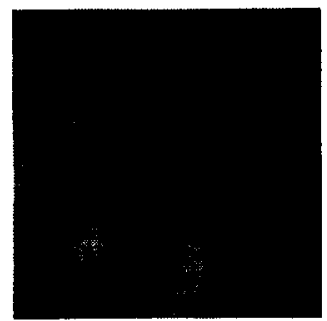

(c)

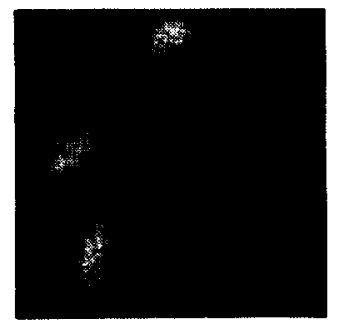

(d)

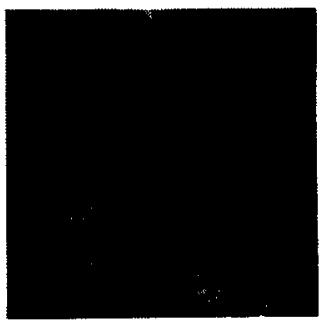

(e) 
Figure 3
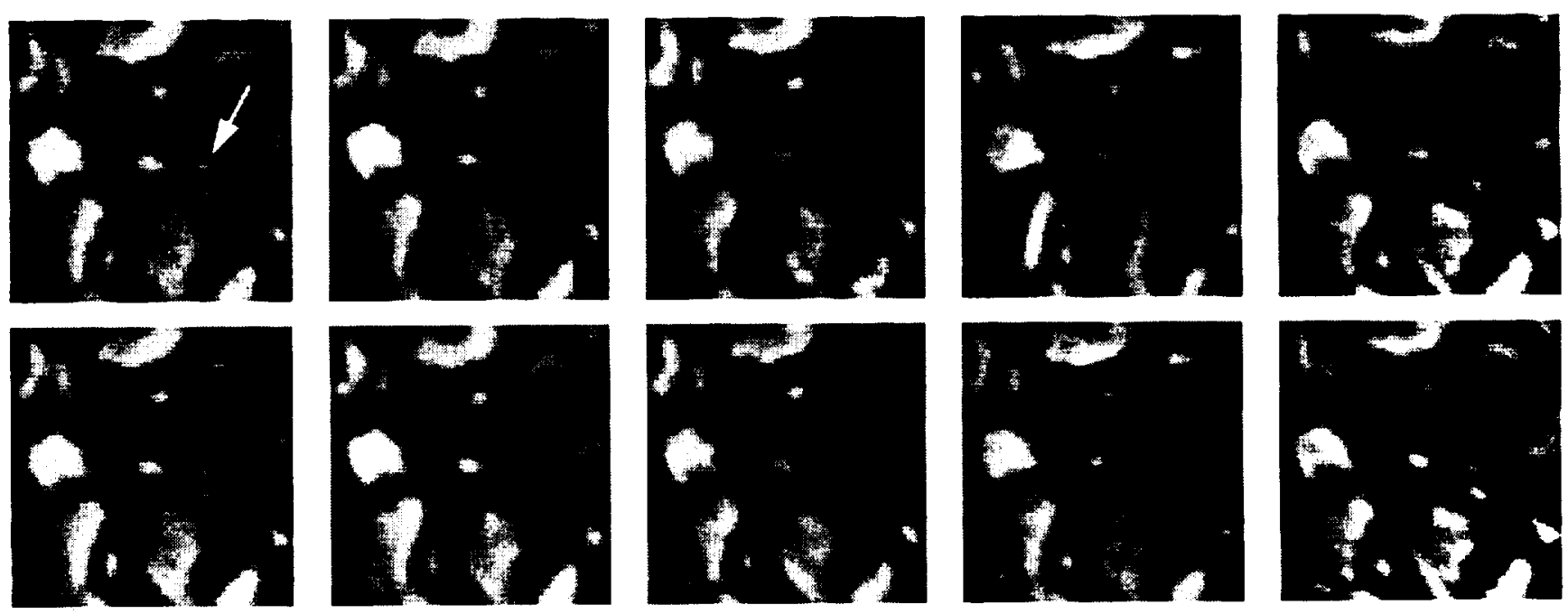

(a)

(b)

(c)

(d)

(e) 
Fyuire 4

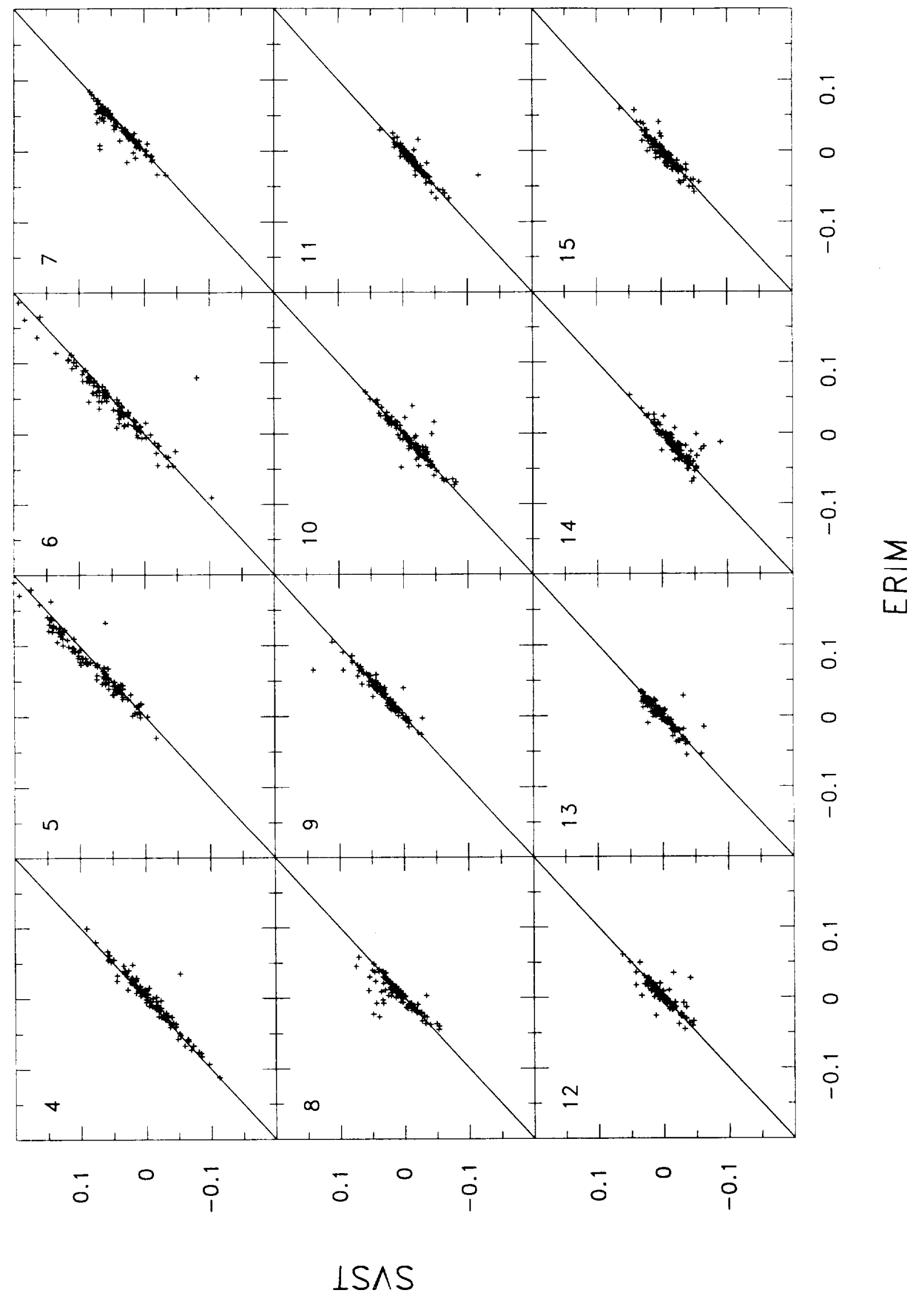


Figire 5

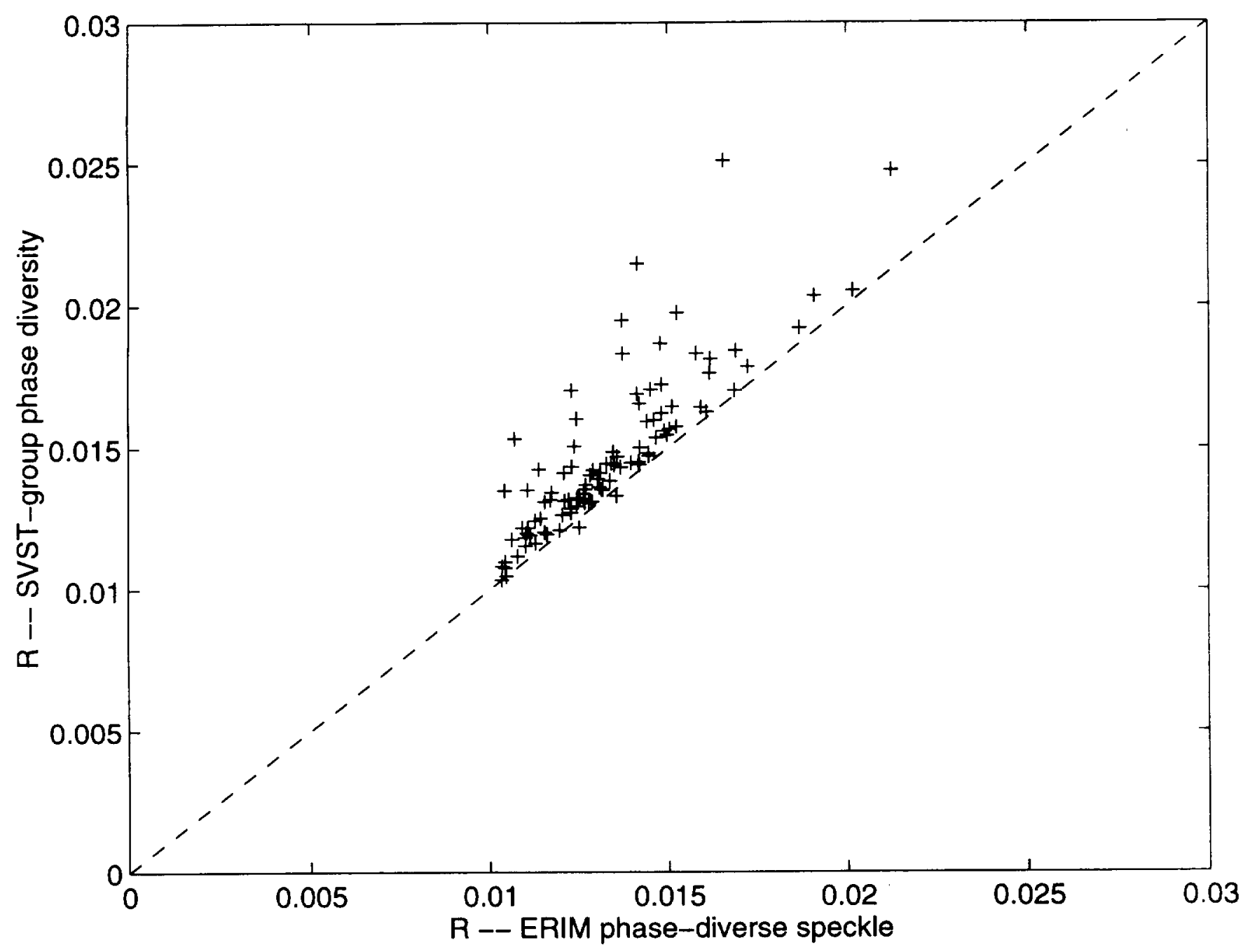


Figure 6

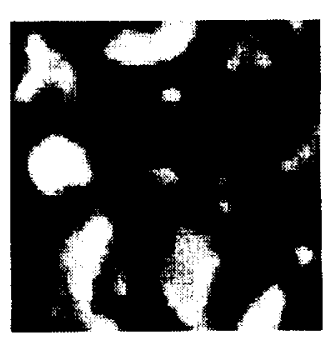

(a)

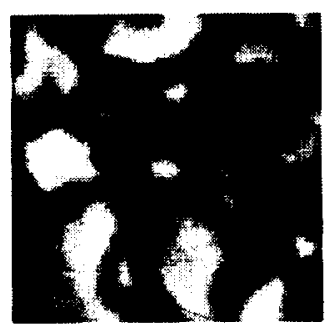

(d)

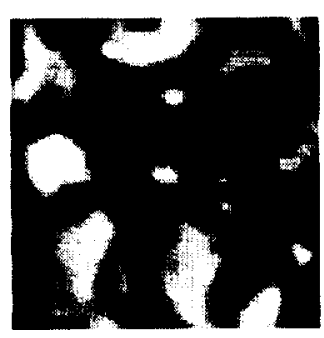

(b)

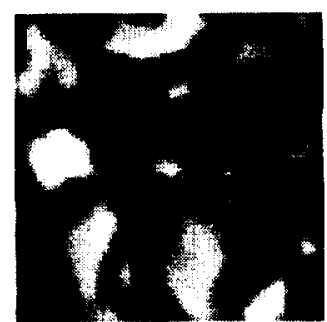

(e)

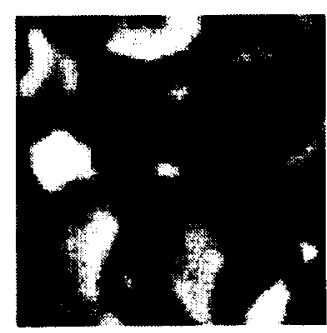

(c)

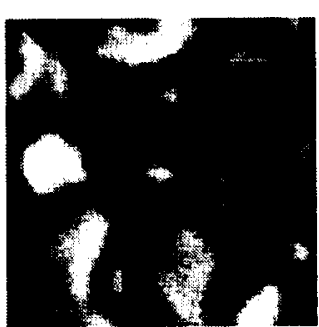

(f) 
Figire 7

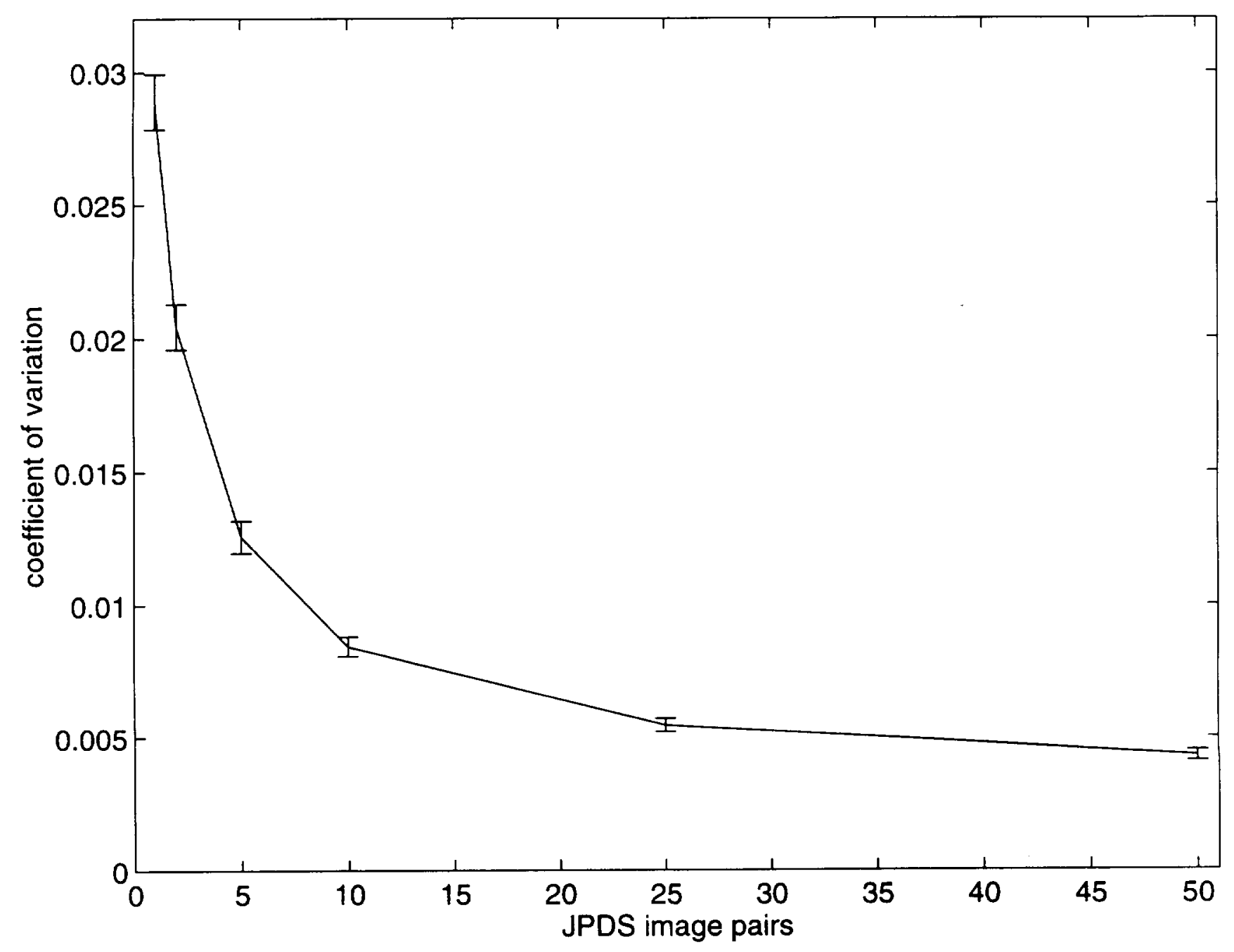


Fyure 8

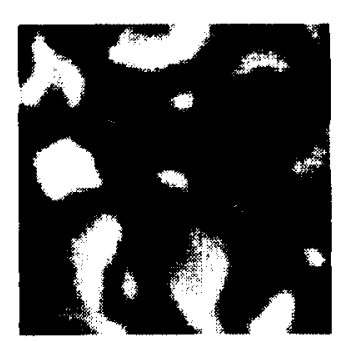

(a)

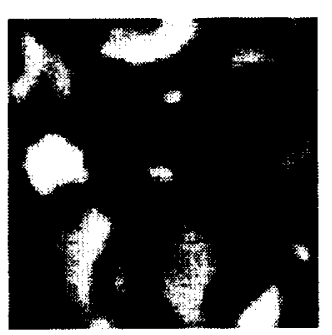

(b)

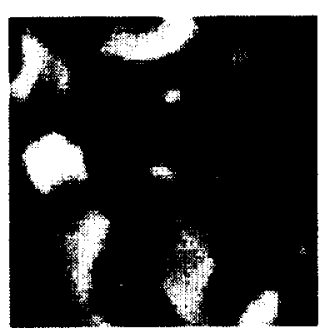

(c)

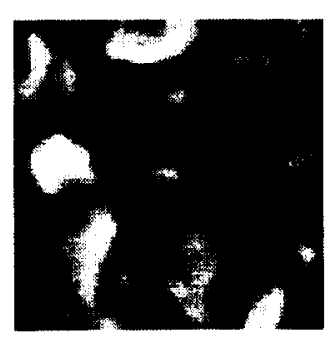

(d)

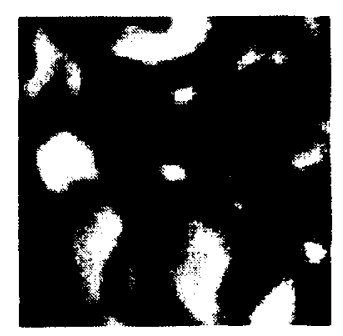

(e) 
Fyire?

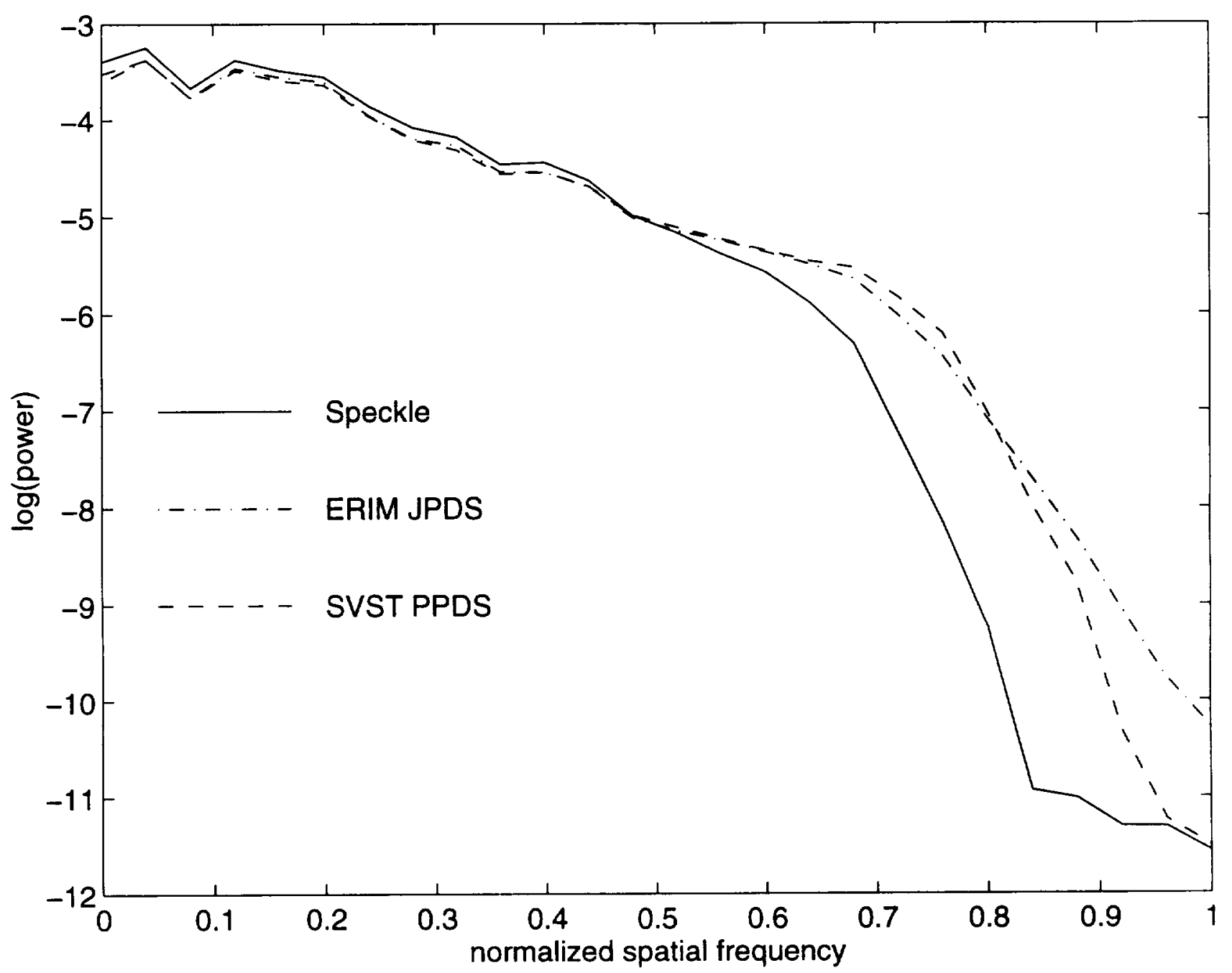


Fiyure $10(a)$

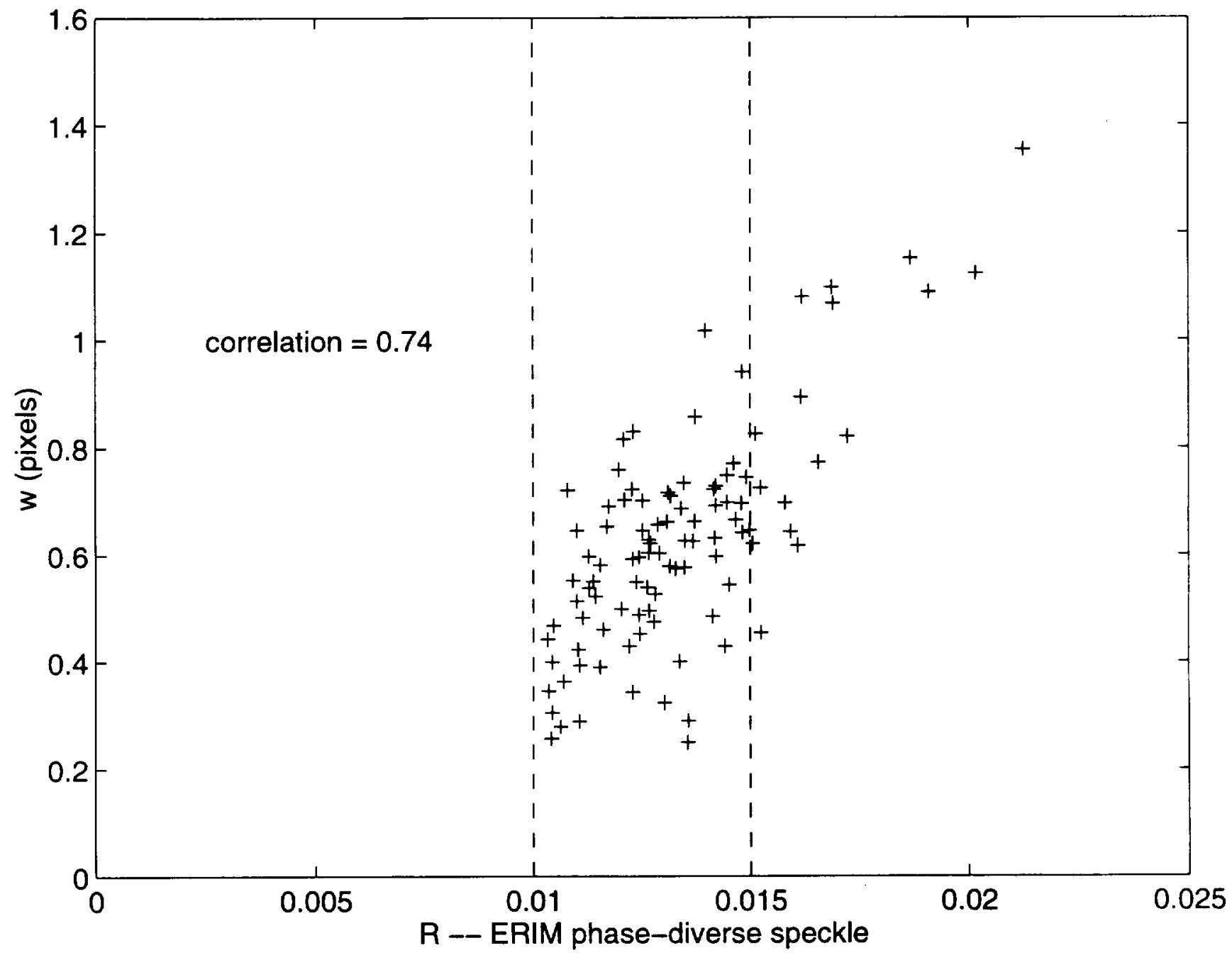

(a) 
Fyuive 1O(b)

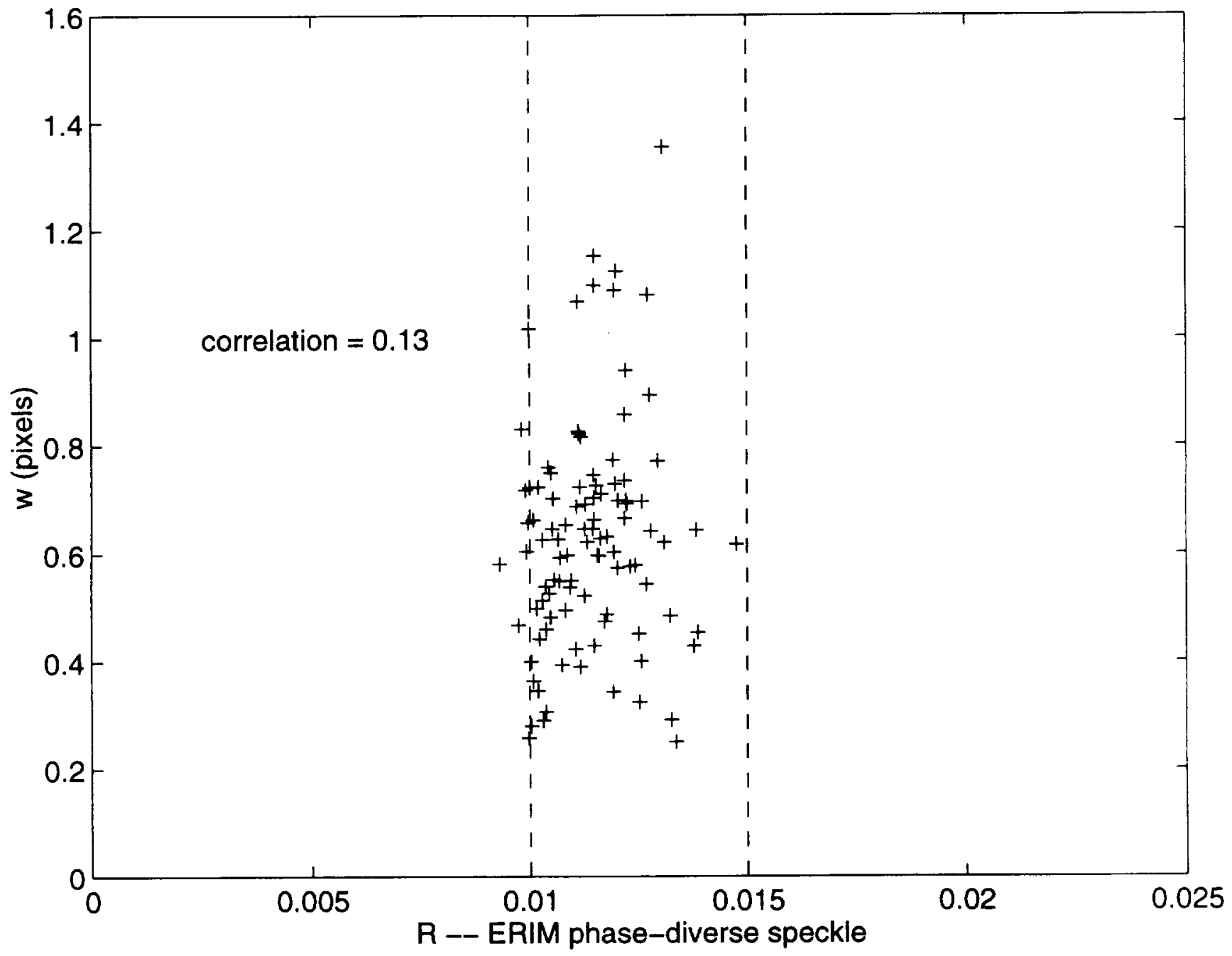

(b) 